\title{
A multiphase model for tissue construct growth in a perfusion bioreactor
}

\author{
R. D. O’Dea, S. L. Waters ${ }^{\dagger} \&$ H. M. Byrne \\ School of Mathematical Sciences, University of Nottingham \\ University Park, Nottingham, NG7 2RD \\ ${ }^{\dagger}$ Mathematical Institute, 24-29 St Giles’, Oxford, OX1 3LB
}

\begin{abstract}
The growth of a cell population within a rigid porous scaffold in a perfusion bioreactor is studied, using a three phase continuum model of the type presented by Lemon et al. (2006) to represent the cell population (and attendant extracellular matrix), culture medium and porous scaffold. The bioreactor system is modelled as a two-dimensional channel containing the cell-seeded rigid porous scaffold (tissue construct) which is perfused with culture medium. The study concentrates on (i) cell-cell and cell-scaffold interactions and, (ii) the impact of mechanotransduction mechanisms on construct composition.

A numerical and analytical analysis of the model equations is presented and, depending upon the relative importance of cell aggregation and repulsion, markedly different cell movement is revealed. Additionally, mechanotransduction effects due to cell density, pressure and shear stress-mediated tissue growth are shown to generate qualitative differences in the composition of the resulting construct. The results of our simulations indicate that this model formulation (in conjunction with appropriate experimental data) has the potential to provide a means of identifying the dominant regulatory stimuli in a cell population.
\end{abstract}

\section{Introduction}

The growth of biological tissue is highly complex, involving the interaction of numerous processes which operate on disparate spatio-temporal scales, from intracellular gene 
networks to tissue-level patterning and mechanics (Peirce et al., 2006). Due to their importance in (for instance) tissue engineering, and various pathological conditions, tissue growth processes have inspired a huge range of theoretical and experimental studies (see Araujo \& McElwain (2004), Cowin (2000, 2004), Curtis \& Riehle (2001) and Sipe (2002) for reviews).

In vitro tissue engineering, which involves creating replacement tissue in the laboratory from a sample of healthy cells or small explants, has the potential to alleviate the chronic shortage of tissue available from donors (Curtis \& Riehle, 2001). Static culture for cell monolayer and small explants has been employed in vitro for many years; however, limitations in the diffusion of nutrients and waste products mean that scale-up to produce constructs of a size appropriate for implant results in the formation of constructs with a viable, proliferating periphery but a necrotic core (Cartmell \& El Haj, 2005). To rectify this, bioreactors, which enable control of the culture environment via circulation/mixing strategies and provision of growth factors and other cell-signalling molecules, are widely used. As well as improving mass transfer, such strategies have a profound effect on the cells' mechanical environment, the consequences of which will be specific to the cell population in question. For instance, fluid flow can have deleterious effects on cartilage regeneration; in contrast, many studies have shown that stimulation via fluid shear stress enhances bone tissue formation (Bakker et al., 2004; Han et al., 2004; Klein-Nulend et al., 1998, 1995b; Weinbaum et al., 1994; You et al., 2000, 2001). Many bioreactors are therefore designed specifically to provide appropriate mechanical stimulation to cell cultures via, e.g. fluid shear stress, or tensile or compressive forces applied on the macroscale or via magnetic particles embedded in the cell membrane (see Cartmell \& El Haj (2005) and Martin et al. (2004) for a review). These stimuli are integrated into the cellular response via a process known as mechanotransduction.

Much research has been concentrated on the study of cartilage and bone tissue regeneration, motivated by the notorious incapacity of the former to self-repair (Lemon \& King, 2007) and the response of the latter to its mechanical environment; an experimental study of bone cell response to mechanical loading provided the inspiration for this research. Advances in the understanding of the mechanisms that regulate tissue growth via experimental or theoretical studies promise to improve the integrity and viability of the resulting tissue constructs; idealised theoretical studies aim to predict optimal protocols for tissue growth, suggest explanations for observed tissue growth phenomena and can provide insights useful in the design of bespoke bioreactor systems.

Studies which consider explicitly the stresses experienced by cells at the microscopic 
level include Jaecques et al. (2004), McGarry et al. (2004) and Tracqui \& Ohayon (2004). The effect of growth-induced (residual) stresses on tissue growth within a macroscale multiphase framework was investigated by Roose et al. (2003), employing a poroelastic model to determine the stress within, and surrounding, a tumour spheroid. Araujo \& McElwain (2005) also presented a general multiphase framework suitable for the consideration of such stresses. Employing a two-phase model, Byrne \& Preziosi (2003a) considered the influence of the cells' environment on their proliferative rate in the context of tumour growth. The tumour was modelled as a viscous fluid phase interacting with an inviscid extracellular fluid. The proliferation of tumour cells was dependent upon nutrient availability (governed by an advection-diffusion equation) and cell density, and a step function was used to switch between two different density and nutrient-dependent responses as the nutrient availability crosses a threshold value. By introducing a parameter associated with the cell's response to growth-induced stress, a critical stress level was predicted, above which the tumour is eliminated. Chaplain et al. (2006) presented a similar model considering tumour cells, normal cells, their associated extracellular matrices (ECM) and a matrix-degrading enzyme. A mollified step function was used to model the transition between the proliferative response of the cells in response to stress. It was shown that reduced contact inhibition or sensitivity to the compressive stress (modelled as proportional to the total tissue volume fraction) leads to elevated proliferation of the tumour cells.

In contrast, O'Dea et al. (2008) neglected the solid characteristics of biological tissue and employed a two-fluid model (representing cells and culture medium; each phase was modelled as a viscous fluid) to investigate the effect of mechanotransduction on tissue construct growth within a perfusion bioreactor. The tissue construct was defined to be the region occupied by the interacting cell and culture medium phases; the remainder of the bioreactor contains only culture medium. Guided by parameter estimation, a simplified model was obtained by exploiting the limit of large interphase viscous drag after Franks (2002) and Franks \& King (2003) in which each phase moves with a common velocity. On the basis of these results, it was concluded that long bioreactors or very low rates of perfusion are required to prevent cells from being flushed out of the bioreactor. For constant tissue growth rate, the model predicted axially-asymmetric construct growth both in static culture conditions and in the presence of perfusion. In static conditions, the upstream periphery of the growing tissue remained fixed whilst tissue growth led to progression of the downstream periphery along the bioreactor. In perfusive culture, both up- and downstream peripheries were advected by the flow; advection of the downstream periphery was augmented by tissue growth. Additionally, pressure and density-dependent cell growth were 
considered and differences between the predicted tissue composition in each case illustrated the potential use of the model to predict the dominant regulatory stimuli in a cell population.

Studies which consider specifically tissue growth in porous scaffolds include Malda et al. (2004) in which the development of oxygen gradients in the absence of perfusion was investigated using a simple diffusion-consumption model. Parameter estimation was achieved via comparison with experimental data. Three-dimensional fluid flow through porous scaffolds in a perfusion bioreactor was studied by Porter et al. (2005) in which a detailed model of a porous scaffold was obtained via micro-computed tomography imaging and the flow profile calculated using the Lattice-Boltzmann method. Relating simulation results to experimental results, it was concluded that a mean pore-surface shear stress of $5 \times 10^{-5} \mathrm{~Pa}$ corresponds to increased cell proliferation and viability. Raimondi (2004) demonstrated that the material properties and cell viability of constructs resulting from perfusion show a two-fold improvement compared to static culture; computational modelling was used to quantify the fluid-dynamical environment at the microscopic level. Modelling of both cell growth and fluid flow within a three-dimensional scaffold in a perfusion bioreactor was considered by Coletti et al. (2006). The flow through the scaffold was governed by Brinkman's equation and nutrient distribution was described by a reaction-advectiondiffusion equation. Cell growth was assumed to depend upon local nutrient availability via an ordinary differential equation.

A weakness of the models presented in e.g. Araujo \& McElwain (2005), Jaecques et al. (2004), McGarry et al. (2004), O'Dea et al. (2008) and Tracqui \& Ohayon (2004) (when considering in vitro tissue growth) is that the polymer scaffold is not treated as a distinct phase; rather, many two phase models assume that it may be modelled within a lumped "cell" phase, and cell-scaffold interactions are necessarily neglected. In this paper, we extend our earlier work (O'Dea et al., 2008) and employ the formulation of Lemon et al. (2006) to derive a three phase model (representing a cell population and attendant ECM, a porous scaffold and a culture medium) which we use to investigate tissue growth in a perfusion bioreactor; however, the formulation is sufficiently general to be applied to a wide range of tissue engineering applications. Such multiphase models (which have been widely used in industrial applied mathematics; e.g. Drew \& Segel (1971)) naturally capture the multiphase nature of tissue growth, enabling explicit modelling of the interactions between the constituent phases, and have therefore been employed in modelling a range of biological phenomena (see, e.g. Byrne \& Preziosi (2003a), Franks \& King (2003), Landman \& Please (2001) and Lemon et al. (2006) and references therein). In common with these 
studies, we represent the cells and associated ECM as a viscous fluid phase that is distinct from the culture medium; the porous scaffold is modelled as a rigid porous medium. The applicability of the model is therefore restricted to tissue constructs whose solid characteristics are dominated by scaffold rigidity. We remark, however, that our generic modelling framework is versatile allowing, for example, elastic or viscoelastic constitutive modelling assumptions for the cell or scaffold phases. Since the cells and ECM are modelled as a single phase, the interactions between the cells and the ECM are neglected; furthermore, the replacement of degrading scaffold by proteoglycan and collagen deposition (for example) is not considered.

We investigate two factors which are of key importance in the growth and adaptation of engineered tissue constructs: (i) cell-cell and cell-scaffold interactions and, (ii) the impact of mechanotransduction mechanisms on construct morphology (specifically, we consider density, pressure and shear stress-mediated tissue growth). As noted above, despite many tissues sharing common mechanotransduction pathways, the influence of the mechanical environment will be specific to the cell population in question. In this paper, we employ our generic modelling framework to investigate a range of biologically-inspired mechanotransduction mechanisms and, in so doing, demonstrate the importance of such effects to in vitro tissue growth and the ability of our model to accommodate a wide variety of such considerations. Conclusions relevant to specific tissue engineering systems may in principle be obtained by modifying the mechanotransduction response functions in line with appropriate experimental data; however, such modifications are beyond the scope of this paper. The influence of perfusion on construct growth is demonstrated by comparing the construct composition resulting from static and perfusive culture conditions. Nutrientlimited growth is not considered here so that we may focus on mechanotransduction. We demonstrate that the relaxation of the large drag assumption and consideration of cellcell and cell-scaffold interactions result in starkly different cell behaviour to that found in O'Dea et al. (2008).

The perfusion bioreactor under consideration is based upon that employed by El-Haj et al. (1990) which comprises a tissue construct within a culture medium-filled cylinder along which a flow is driven (see figure 1). The bioreactor is designed to allow cells seeded in a porous scaffold to be subjected to perfusion with media and direct compression using a piston. The cell-seeded scaffold has a mean porosity of 97\% (Freyria et al., 2005); perfusion is effected using a peristaltic pump at a rate of $0.1 \mathrm{ml} / \mathrm{min}$. This system has been employed to investigate the influence of perfusion and macroscale compression on tissue growth; in this study, we concentrate on the effect of perfusion and neglect the macroscale 
forcing provided by the piston.

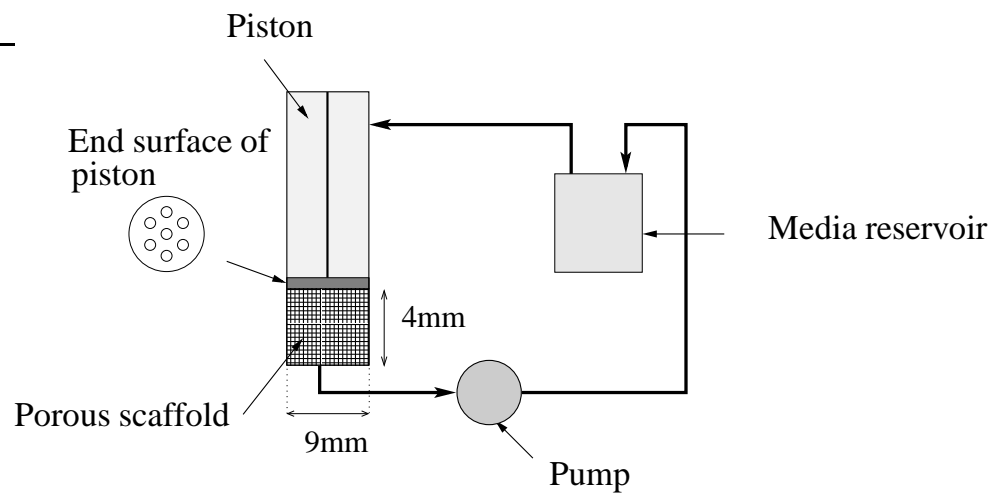

Figure 1: The bioreactor system of El-Haj et al. (1990).

The structure of this paper is as follows. In $\S 2$ we present the three-phase governing equations; employing the long-wavelength limit (assuming that the bioreactor is long and thin) and considering constant, spatially-homogeneous scaffold porosity, we reduce the system to a pair of differential equations governing the cell phase volume fraction and the culture medium pressure, together with appropriate boundary conditions. Solutions to these equations are presented in $\S \S 3$ and 4 .

In $\S 3$, uniform growth is considered; numerical simulations are presented in $\S 3.1$ and validated against analytic solutions in the limit for which the cell volume fraction is asymptotically small (§3.2). In $\S 3.3$, the influence of cell-cell and cell-scaffold interactions on tissue growth is investigated further by considering simplified functional forms for these effects. Lastly, in $\S 4$, we consider mechanotransduction-affected growth, studying the response of a cell population to the local density, pressure and shear stress. A discussion of the model results and their applications within the field of tissue engineering is given in $\S 5$, together with suggestions for further work.

\section{A three phase model for tissue construct growth}

We develop a three phase model relevant to tissue engineering processes, employing the general multiphase formulation given in Lemon et al. (2006) and Lemon \& King (2007). For brevity we do not present the derivation in detail: the reader is directed to Drew (1983), Kolev (2002), Lemon et al. (2006) and O'Dea (2008) for a more comprehensive discussion of multiphase modelling considerations. 
We consider the growth of a tissue construct within a nutrient-rich fluid culture medium and investigate the effect of cell-cell and cell-scaffold interactions, as well as that of mechanotransduction, on the growth of a tissue construct within a perfusion bioreactor. The bioreactor under consideration is based upon a system employed by El-Haj et al. (1990) (see §1) which we represent as a two-dimensional channel containing a mixture of interacting phases. A two-dimensional channel geometry is employed for simplicity; however, generalisation to a cylindrical geometry is straightforward. The multiphase mixture comprises two viscous fluids and one rigid, porous phase. The cells and ECM, which are represented by a single phase (henceforth denoted the "cell phase"), and culture medium are modelled as viscous fluids, and the remaining rigid phase represents the scaffold. Tissue growth is represented by an increase in cell phase volume fraction, corresponding to the combined effects of cell proliferation and ECM deposition. Viscous fluid-based models for biological tissue growth have been widely used (see e.g. Byrne et al. (2003), Byrne \& Preziosi (2003b), Franks (2002) and Franks \& King (2003)); such models are appropriate when the timescale of elastic relaxation is short in comparison to that of growth (Bittig et al., 2008; Franks \& King, 2003; King \& Franks, 2004). Perfusion is represented by a pressure-driven flow of culture medium.

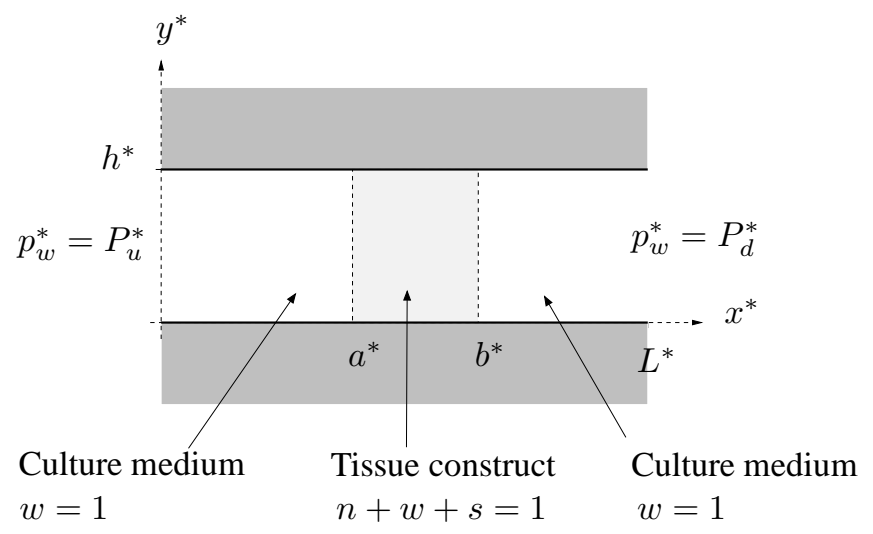

Figure 2: Definition sketch: a two-dimensional channel of length $L^{*}$ and width $h^{*}$ containing a construct of length $b^{*}-a^{*}$.

A Cartesian coordinate system $\boldsymbol{x}^{*}=\left(x^{*}, y^{*}\right)$ is chosen with corresponding coordinate directions $(\hat{\boldsymbol{x}}, \hat{\boldsymbol{y}})$ and the channel occupies $0 \leqslant x^{*} \leqslant L^{*}, 0 \leqslant y^{*} \leqslant h^{*}$. In this paper, asterisks distinguish dimensional quantities from their dimensionless equivalents. We associate with the cell, culture medium and scaffold phases a volume fraction denoted, $n, w, s$, respectively, a volume-averaged velocity, $\boldsymbol{u}_{i}^{*}=\left(u_{i}^{*}, v_{i}^{*}\right)$, pressure, $p_{i}^{*}$ and stress 
tensor $\boldsymbol{\sigma}_{i}$ (where $i=n, w, s$ denotes variables associated with each phase) and assume that these are functions of $\boldsymbol{x}^{*}$ and $t^{*}$, where $t^{*}$ represents time.

For convenience, we confine the tissue construct to the region $a^{*} \leqslant x^{*} \leqslant b^{*}$ in which $s>0$ (see figure 2), stipulating that the cell phase must remain confined within the scaffold. We achieve this by imposing a no-flux boundary condition on the cell phase at the scaffold edge. Formulating the problem in this way allows us to simplify the governing equations in the up- and downstream regions, whilst retaining the full complexity of the three phase system within the construct region. The problem may be solved separately in each region, and the solutions coupled together via appropriate conditions.

The multiphase model takes the form of mass and momentum balances for each phase, together with appropriate constitutive laws. Neglecting inertial effects and assuming that each phase is incompressible with the same density, the equations governing the $i^{\text {th }}$ phase (with volume fraction $\phi_{i}$ ) are as follows (see Lemon et al. (2006)):

$$
\begin{array}{ll}
\text { conservation of mass: } & \frac{\partial \phi_{i}}{\partial t^{*}}+\nabla^{*} \cdot\left(\phi_{i} \boldsymbol{u}_{i}^{*}\right)=S_{i}^{*}+D^{*} \nabla^{* 2} \phi_{i} ; \\
\text { conservation of momentum: } & \nabla^{*} \cdot\left(\phi_{i} \boldsymbol{\sigma}_{i}^{*}\right)+\sum_{j \neq i} \mathbf{F}_{i j}^{*}=\mathbf{0},
\end{array}
$$

in which $S_{i}^{*}$ is the net material production term associated with phase $i$; so that mass is conserved, we assume $\sum S_{i}^{*}=0 . \mathbf{F}_{i j}^{*}$ is the force exerted by phase $j$ on phase $i$ which obeys $\mathbf{F}_{i j}^{*}=-\mathbf{F}_{j i}^{*}$. Conservation conditions may be obtained by summing equations (2.1) and (2.2) over all phases and exploiting the no-voids condition: $\sum \phi_{i}=1$.

We remark that a diffusive term has been added to the mass conservation equation (2.1) and for simplicity the diffusivity of each phase is assumed to be equal; whilst cells do exhibit random motion, in this model the growth and flow-driven velocity field is the dominant mechanism giving rise to cell movement and diffusive terms are expected to be negligible (Franks et al., 2003; King \& Franks, 2004). However, we retain these terms for numerical convenience since they eliminate the moving boundaries between the tissue construct and culture medium, ensuring that we need not track explicitly the sharp interface which is evident when $D^{*}=0$.

We now pause to discuss the constitutive modelling assumptions. We model the scaffold as a rigid porous phase and, for simplicity, assume that its volume fraction is spatiallyhomogeneous and constant in time. Equation (2.1) is therefore redundant for this phase and the no-voids condition becomes $n+w=\theta$, where $\theta=1-s$ is the porosity of the scaffold; in what follows, it is more convenient to work in terms of $n$ and $\theta$. The cell and culture medium phases are modelled as viscous fluids and we therefore employ the stan- 
dard viscous stress tensors for these phases (with dynamic and bulk viscosities $\mu_{i}^{*}, \lambda_{i}^{*}$; $i=n, w)$. For consistency, we choose the same form for $\boldsymbol{\sigma}_{s}$, taking the limit $\mu_{s}^{*} \rightarrow \infty$, $\boldsymbol{u}_{s} \rightarrow \mathbf{0}$.

The interphase forces $\mathbf{F}_{i j}^{*}$ comprise contributions from interphase viscous drag (which is assumed to be proportional to the volume fraction of each phase and their relative velocity) and active forces arising between the phases. We assume that the cell phase generates an intraphase pressure, $\Sigma_{n}^{*}$, resulting from interactions within the cell phase such as osmotic stresses or surface tension within cell membranes. Additionally, tractions between the cell and scaffold phases give rise to an additional pressure contribution, $\psi_{n s}^{*}$ (see Lemon et al. (2006) for more details). Assuming that interactions between the culture medium and scaffold phases are limited to viscous drag we find that the pressure in the cell phase is related to that in the culture medium via:

$$
p_{n}^{*}=p_{w}^{*}+\Sigma_{n}^{*}+(1-\theta) \psi_{n s}^{*}
$$

and the interphase forces are given by:

$$
\begin{aligned}
& \mathbf{F}_{n w}^{*}=\theta p_{w}^{*} \nabla^{*} n+k^{*} n w\left(\boldsymbol{u}_{w}^{*}-\boldsymbol{u}_{n}^{*}\right)=-\mathbf{F}_{w n}^{*}, \\
& \mathbf{F}_{s w}^{*}=p_{w}^{*}(1-\theta) \nabla^{*} n+k^{*}(\theta-n)(1-\theta) \boldsymbol{u}_{w}^{*}=-\mathbf{F}_{w s}^{*}, \\
& \mathbf{F}_{n s}^{*}=\left(p_{w}^{*}+\psi_{n s}^{*}\right)(1-\theta) \nabla^{*} n-k^{*} n(1-\theta) \boldsymbol{u}_{n}^{*}=-\mathbf{F}_{s n}^{*},
\end{aligned}
$$

where $k^{*}$ is the coefficient of viscous drag which is assumed to be constant. The interphase interaction terms $\Sigma_{n}^{*}$ and $\psi_{n s}^{*}$ and the material production rates (accommodating a range of tissue growth processes) will be specified once the model has been cast in dimensionless form.

We non-dimensionalise as follows:

$$
\left.\begin{array}{l}
\boldsymbol{x}^{*}=L^{*} \boldsymbol{x}, t^{*}=t / K_{m}^{*}, \boldsymbol{u}_{i}^{*}=K_{m}^{*} L^{*} \boldsymbol{u}_{i}, S_{i}^{*}=K_{m}^{*} S_{i}, \\
\left(p_{i}^{*}, \Sigma_{n}^{*}, \psi_{n s}^{*}\right)=K_{m}^{*} \mu_{w}^{*}\left(p_{i}, \Sigma_{n}, \psi_{n s}\right)
\end{array}\right\}
$$

where $K_{m}^{*}$ is a typical tissue growth rate and the channel now occupies $0 \leqslant x \leqslant 1$, $0 \leqslant y \leqslant h=h^{*} / L^{*}$; the length of the construct is $a \leqslant x \leqslant b$, where $(a, b)=\left(a^{*}, b^{*}\right) / L^{*}$. A viscous scaling is employed for the pressure in each phase $\left(p_{i}^{*}\right)$ since we assume that viscous effects dominate inertia. The timescale of interest is that of tissue growth; under perfusion, the flow rate and dimensions of the bioreactor system of El-Haj et al. given in $\S 1$ indicate a flow timescale of approximately 2.5 minutes. This is short in comparison to the timescale of tissue growth (employing this system, Wood et al. (2006) subjected cells to perfusion for one week); however, we consider the ratio of the growth and flow timescales 
to be of $\mathcal{O}(1)$ both in static (in which the flow is a consequence of tissue growth only) and perfusive culture conditions, employing fast growth rates to minimise computation time and to illustrate features of the system.

In dimensionless form, the model equations are:

$$
\begin{aligned}
& \frac{\partial n}{\partial t}+\nabla \cdot\left(n \boldsymbol{u}_{n}\right)=S_{n}+D \nabla^{2} n, \\
& \nabla \cdot\left(n \boldsymbol{u}_{n}+(\theta-n) \boldsymbol{u}_{w}\right)=0 ; \\
& (\theta-n) \nabla p_{w}+k n(\theta-n)\left(\boldsymbol{u}_{w}-\boldsymbol{u}_{n}\right)+k(1-\theta)(\theta-n) \boldsymbol{u}_{w}- \\
& \nabla \cdot\left[(\theta-n)\left(\nabla \boldsymbol{u}_{w}+\nabla \boldsymbol{u}_{w}^{T}\right)+\gamma_{w}(\theta-n) \nabla \cdot \boldsymbol{u}_{w} \mathbf{I}\right]=\mathbf{0}, \\
& \begin{array}{r}
\nabla \cdot\left[-\left(\theta p_{w}+n \Sigma_{n}+n(1-\theta) \psi_{n s}\right) \mathbf{I}+\mu_{n} n\left(\nabla \boldsymbol{u}_{n}+\nabla \boldsymbol{u}_{n}^{T}\right)+\right. \\
\left.\gamma_{n} n \nabla \cdot \boldsymbol{u}_{n} \mathbf{I}+(\theta-n)\left(\nabla \boldsymbol{u}_{w}+\nabla \boldsymbol{u}_{w}^{T}\right)+\gamma_{w}(\theta-n) \nabla \cdot \boldsymbol{u}_{w} \mathbf{I}\right]+ \\
\quad \nabla n(1-\theta) \psi_{n s}-k n(1-\theta) \boldsymbol{u}_{n}-k(\theta-n)(1-\theta) \boldsymbol{u}_{w}=\mathbf{0} .
\end{array}
\end{aligned}
$$

Equations (2.8) and (2.9) are statements of conservation of mass for the cell phase and the multiphase mixture; equation (2.10) expresses conservation of momentum for the culture medium and (2.11) is the momentum equation for the two phase mixture of cells and culture medium. We employ this equation in preference to the momentum equation for the three phase mixture for convenience (see Lemon \& King (2007)). Assuming that the scaffold porosity is constant in space and time enabled significant simplification of the three-phase governing equations, the rigid scaffold phase only appearing via the constant porosity, $\theta$, and the cell-scaffold interactions.

The dimensionless parameters $D, \mu_{n}, k, \gamma_{w}$ and $\gamma_{n}$ are defined:

$$
D=\frac{D^{*}}{K_{m}^{*} L^{*}}, \quad \mu_{n}=\frac{\mu_{n}^{*}}{\mu_{w}^{*}}, \quad k=\frac{k^{*} L^{* 2}}{\mu_{w}^{*}}, \quad \gamma_{w}=\frac{\lambda_{w}^{*}}{\mu_{w}^{*}}, \quad \gamma_{n}=\frac{\lambda_{n}^{*}}{\mu_{w}^{*}} .
$$

The physical interpretation of the dimensionless diffusion coefficient (or inverse Peclet number) $D$, relative viscosity $\mu_{n}$, and drag coefficient $k$ is self-evident. The parameter $\gamma_{i}$ describes the relative importance of the viscosity associated with the rate of change of volume of the $i^{\text {th }}$ phase compared to that associated with fluid shear. It is usual to take $\lambda_{i}^{*}=-2 \mu_{i}^{*} / 3$ implying $\gamma_{w}=-2 / 3$ and $\gamma_{n}=-2 \mu_{n}^{*} / 3 \mu_{w}^{*}$ (Franks, 2002; Franks \& King, 2003; King \& Franks, 2004; Lemon et al., 2006) so that in the viscous stress tensor we have $p_{i}=-\sigma_{i, k k} / 3$. 
Appropriate boundary conditions on this problem are as follows:

$$
\begin{aligned}
& \frac{\partial n}{\partial y}=0, \quad \boldsymbol{u}_{w}=\mathbf{0}=\boldsymbol{u}_{n}, \quad \text { on } y=0, h, \\
& p_{w}=P_{u}, \quad v_{w}=0, \quad \text { on } x=0, \\
& p_{w}=P_{d}, \quad v_{w}=0, \quad \text { on } x=L,
\end{aligned}
$$

where the dimensionless up- and downstream pressures are defined:

$$
P_{u}=\frac{P_{u}^{*}}{K_{m}^{*} \mu_{w}^{*}}, \quad P_{d}=\frac{P_{d}^{*}}{K_{m}^{*} \mu_{w}^{*}} .
$$

Equations (2.13) guarantee no-penetration and no-slip at $y=0, h$ and equations (2.14) and (2.15) set an axial pressure drop which drives a (unidirectional) flow. In the case of static culture conditions, we choose $P_{u}=P_{d}=0$ without loss of generality. Conditions on $n$ at $x=0, L$ are not required since the cells are confined to $a \leqslant x \leqslant b$.

It remains to specify the functions $\psi_{n s}$ and $\Sigma_{n}$, whose definition, together with appropriate material transfer terms, $S_{i}(\boldsymbol{x}, t)$, and initial conditions, completes our model formulation. Following Breward et al. (2002), Byrne et al. (2003) and Lemon et al. (2006), appropriate expressions are taken to be

$$
\Sigma_{n}=n\left(-\nu+\frac{\delta_{a} n}{\theta-n}\right), \quad \psi_{n s}=-\chi+\frac{\delta_{b} n}{\theta-n},
$$

for constants $\nu, \delta_{a}, \chi, \delta_{b}>0$. The first term in each of these expressions reflects the cells' tendency to aggregate at low densities and their affinity for the scaffold, respectively. The second term represents the repulsive forces between cells and between the cells and scaffold which arise when they come into close contact (Lemon et al., 2006). Initial conditions will be specified in $\S 3$ when numerical solution of the model equations is undertaken.

The relevance of this formulation to tissue growth processes hinges upon the appropriate choice of material transfer terms, $S_{i}(\boldsymbol{x}, t)$. The growth of the tissue construct will be strongly influenced by the cells' mechanochemical environment and we therefore consider the influence of cell density, pressure and shear stress on the evolution and eventual composition of the tissue construct, corresponding to $S_{n}(n), S_{n}\left(p_{n}\right)$ and $S_{n}(\tau)$, where $\tau$ denotes the flow-induced shear stress. The choice $S_{n}(n)$ enables us to capture the effect of contact inhibition (Chaplain et al., 2006) and tissue growth-induced stress (Fung, 1991; Roose et al., 2003) on cell behaviour. An alternative way to model the effect of local density on cell behaviour is to consider the pressure of the cell phase as an indicator of cell density; since $p_{n}$ is intimately connected to the pressure of the culture medium, this choice has the added advantage of including the response of cells to the local fluid 
dynamics. The response of cells to culture medium pressure is well documented, especially with respect to bone tissue growth; for example, many authors have shown that bone cells respond to intermittent hydrostatic compression with bone resorption inhibited and bone formation stimulated (Klein-Nulend et al. (1995a) and references therein), and increased adhesion (Haskin et al., 1993) and osteopontin (a protein implicated in the bone remodelling process) expression (Owan et al., 1997). Excessively high hydrostatic pressure $(>200 \mathrm{kPa}$ ) has been shown to exert an inhibitory effect on bone-specific gene expression (Roelofsen et al., 1995). Similarly, many studies have reported that bone cells are highly sensitive to stimulation via flow-induced shear stress; indeed, many theoretical and experimental studies propose fluid shear stress as the dominant regulatory mechanism for in vivo bone tissue remodelling (Bakker et al., 2004; Han et al., 2004; Klein-Nulend et al., 1998, 1995b; Weinbaum et al., 1994; You et al., 2000, 2001). Functional forms for the rates $S_{n}(n), S_{n}\left(p_{n}\right)$ and $S_{n}(\tau)$ will be specified subsequently.

\subsection{Long wavelength limit}

We simplify the governing equations (2.8)-(2.11) by considering the limit for which the aspect ratio of the channel is small, corresponding to $h \ll 1$. We rescale via:

$$
y=h \hat{y}, \quad v_{i}=h \hat{v}_{i}, \quad p_{w}=\hat{p}_{w} / h^{2}, \quad \Sigma_{n}=\hat{\Sigma}_{n} / h^{2}, \quad \psi_{n s}=\hat{\psi}_{n s} / h^{2},
$$

and the channel now occupies $0 \leqslant x \leqslant 1,0 \leqslant \hat{y} \leqslant 1$. Additionally, the dimensionless values $x=a, b$ must now obey $a, b-a, 1-b \gg h$.

The rescaling of the intraphase pressure and interphase traction functions, which ensures that cell-cell and cell-scaffold interactions are retained at leading order, implies $\left(\nu, \delta_{a}, \chi, \delta_{b}\right)=\left(\hat{\nu}, \hat{\delta}_{a}, \hat{\chi}, \hat{\delta}_{b}\right) / h^{2}$; the remaining parameters $k, \mu_{n}, \gamma_{w}$ and $\gamma_{n}$ are $\mathcal{O}(1)$. In this limit, the viscosity associated with the rate of change of volume of each phase, as well as the interphase viscous drag terms are neglected from the momentum equations (2.10) and (2.11) at leading order. Dropping the carets for brevity, we deduce that, at leading order, the pressure $\left(p_{w}\right.$ and $\left.p_{n}\right)$ and the volume fraction ( $w$ and $\left.n\right)$ of each phase are functions of $x$ and $t$ only and the flow is unidirectional. The axial velocities $u_{w}$ and $u_{n}$ are given by:

$$
u_{w}=\frac{1}{2} \frac{\partial p_{w}}{\partial x} y(y-1), \quad u_{n}=\frac{1}{2 \mu_{n}} \frac{\partial \bar{p}}{\partial x} y(y-1),
$$

where the lumped pressure $\bar{p}(x, t)$ is defined:

$$
\frac{\partial \bar{p}}{\partial x}=\frac{\partial p_{w}}{\partial x}+\frac{1}{n} \frac{\partial}{\partial x}\left(n \Sigma_{n}\right)+(1-\theta) \frac{\partial \psi_{n s}}{\partial x} .
$$


The solution for the culture medium velocity $(2.19 a)$ is valid throughout the channel; exterior to the region $a \leqslant x \leqslant b$, we have $n=0, \theta=1, \boldsymbol{u}_{n}=\mathbf{0}$. Averaging the conservation of mass equations across the channel and employing the no-penetration condition at the channel wall, we may now express the model as a pair of coupled differential equations for $n(x, t)$ and $p_{w}(x, t)$. We obtain:

$$
\begin{gathered}
\frac{\partial n}{\partial t}+\frac{1}{12} \frac{\partial}{\partial x}\left\{(\theta-n) \frac{\partial p_{w}}{\partial x}\right\}=S_{n}+D \frac{\partial^{2} n}{\partial x^{2}} \\
\frac{\partial^{2} p_{w}}{\partial x^{2}}+\frac{\bar{\mu}}{\bar{\mu} n+\theta} \frac{\partial n}{\partial x} \frac{\partial p_{w}}{\partial x}=-\frac{1}{\mu_{n}(\bar{\mu} n+\theta)}\left[\frac{\partial^{2}\left(n \Sigma_{n}\right)}{\partial x^{2}}+(1-\theta) \frac{\partial}{\partial x}\left(n \frac{\partial \psi_{n s}}{\partial x}\right)\right],
\end{gathered}
$$

in which $\bar{\mu}=1 / \mu_{n}-1 \leqslant 0$ and $S_{n}(x, t)$ denotes the averaged material transfer rate for the cell phase. For convenience we have employed the mass conservation equation for the culture medium phase in place of (2.8); equation (2.22) is obtained by averaging the total conservation of mass equation (2.9). For pressure-independent material transfer (e.g. $S_{n}=S_{n}(n)$ ), this system may be reduced to an equation for $n$ by taking a first integral of (2.22) to obtain an expression for the advection term $\partial p_{w} / \partial x$. Equations (2.21) and (2.22) are to be solved in the region $a \leqslant x \leqslant b$; in the following, we establish appropriate boundary conditions to apply at $x=a, b$.

\subsubsection{Boundary conditions}

Boundary conditions at $x=0,1$ are given by (2.14) and (2.15); we now derive appropriate conditions to apply at $x=a, b$. Imposing continuity of flux and normal stress across the two boundaries $x=a, b$, we obtain the following jump conditions:

$$
\begin{aligned}
{\left[\left\langle u_{w}\right\rangle\right]^{-} } & =\left[n\left\langle u_{n}\right\rangle+(\theta-n)\left\langle u_{w}\right\rangle\right]^{+} \\
{\left[p_{w}\right]^{-} } & =\left[n p_{n}+(\theta-n) p_{w}\right]^{+}
\end{aligned}
$$

where $\langle\cdot \cdot\rangle=\int_{0}^{1} \cdots \mathrm{d} y$ denotes averaging across the channel and $p_{n}$ is given by the dimensionless version of (2.3). The superscript ' + ' indicates the limiting value $x=a$ (or $b$ ) from within $a \leqslant x \leqslant b$ and ' - ' denotes the limiting value from the exterior. An additional condition governing the behaviour of the cell volume fraction at $x=a, b$ may be derived by requiring that the cell phase be confined within the scaffold. Noting from (2.8) that the averaged flux of cells is $\langle\mathrm{J}(\boldsymbol{x}, t)\rangle=n\left\langle u_{n}\right\rangle-D \partial n / \partial x$, we find that no efflux of cells from the region $a \leqslant x \leqslant b$ is assured if $n$ obeys:

$$
n\left\langle u_{n}\right\rangle=D \frac{\partial n}{\partial x} \quad \text { on } \quad x=a, b .
$$


Considering equation (2.22) in the absence of cells and scaffold, it is straightforward to show that continuity of total flux requires that the culture medium pressure in the regions exterior to the tissue construct is linear with the same gradient:

$$
p_{w}(x, t)= \begin{cases}A(t) x+P_{u} & 0 \leqslant x<a \\ A(t)(x-1)+P_{d} & b<x \leqslant 1\end{cases}
$$

In view of (2.19) and (2.26), the conditions (2.23)-(2.25) imply:

$$
\begin{aligned}
& p_{w}=\frac{A a+P_{u}-n \Sigma_{n}-(1-\theta) n \psi_{n s}}{\theta}, \quad \frac{\partial p_{w}}{\partial x}=\frac{A+12 D \frac{\partial n}{\partial x}}{\theta-n}, x=a,(2.27 a, b) \\
& p_{w}=\frac{A(b-1)+P_{d}-n \Sigma_{n}-(1-\theta) n \psi_{n s}}{\theta}, \frac{\partial p_{w}}{\partial x}=\frac{A+12 D \frac{\partial n}{\partial x}}{\theta-n}, x=b, \quad(2.28 a, b) \\
& \frac{\partial n}{\partial x}=\frac{1}{12 D} \frac{n-\theta}{n+\mu_{n}(n-\theta)}\left\{\frac{A n}{\theta-n}+\frac{\partial\left(n \Sigma_{n}\right)}{\partial x}+(1-\theta) n \frac{\partial \psi_{n s}}{\partial x}\right\}, x=a, b .
\end{aligned}
$$

Equations (2.27) and (2.28) provide four conditions on $p_{w}$, two of which may be specified as boundary conditions, the remaining conditions serving as constraints on the function, $A(t)$. The apparent overspecification of $A(t)$ is due to the imposition of continuity of total flux which demands that the up- and downstream pressure gradients are equal. Either of the remaining conditions may therefore be used to specify $A(t)$. In the proceeding analysis, we choose to impose equations (2.27a) and (2.28a) as boundary conditions and use (2.27b) to determine $A(t)$. The fourth condition (2.28b) is employed as an additional accuracy check in the following numerical scheme, ensuring that continuity of flux is obeyed.

In the following sections, we investigate the effect of (i) interactions between cells and between cells and the scaffold, and (ii) the mechanical environment, on the growth of a tissue construct. In $\S 3$, we consider uniform growth. Numerical solutions presented in $\S 3.1$ are validated by studying the model equations in the limit for which the cell volume fraction is asymptotically small ( $\S 3.2)$, a limit for which analytic solutions may be constructed. In $\S 3.3$, the influence of intraphase pressure and interphase traction on cell behaviour is demonstrated by considering simplified functional forms for these effects. In $\S 4$, we further extend the model by postulating functional forms for the material transfer rate, $S_{n}$, which allow the influence of a range of mechanical stimuli on the growth response of the cells to be investigated. 


\section{Solution: uniform growth}

\subsection{Numerical solution}

We first consider uniform growth, in which case the rates of tissue construct growth and death are constant so that

$$
S_{n}=-S_{w}=\left(k_{m}-k_{d}\right) n,
$$

wherein the dimensionless parameter $k_{m}$ represents the combined rate of cell proliferation and ECM deposition, whilst $k_{d}$ represents the combined rate of cell death and ECM degradation. These parameters are related to the corresponding dimensional rates via $k_{i}=k_{i}^{*} / K_{m}^{*}$ and are assumed to be $\mathcal{O}(1)$. We remark here that in all of the subsequent numerical simulations, the parameter values are selected to illustrate the behaviour of the model under a particular growth regime; the chosen values are given in the relevant figure captions.

To illustrate the behaviour of the model, we consider the following initial cell distribution:

$$
n(x, 0)=0.1[\tanh (75(x-0.45))-\tanh (75(x-0.55))],
$$

representing a small population of cells distributed in the axial centre of the channel (at $x=0.5$ ): we arbitrarily choose $a=0.25, b=0.75$. The influence of alternative initial cell seedings will be investigated in a subsequent study.

Equation (2.21) subject to (2.29) is solved using a semi-implicit predictor-corrector time-stepping method (Peregrine, 1967), and the corresponding culture medium pressure is calculated using (2.22), (2.27a) and (2.28a). A shooting method is used to calculate $A(t)$ at each time-step via the constraint (2.27b): $p_{w}$ is calculated using an initial guess for $A$; the error is then calculated using $(2.27 b)$ and a new value chosen according to a simple bisection routine if the error is too large. Lastly, continuity of flux is checked using equation (2.28b). The NAG routines DGETRI, DGETRF and DGETRS are employed in this numerical scheme; DGETRI performs the matrix inversion required in the re-meshing routine and DGETRS solves the linear systems associated with equations (2.21) and (2.22), using the LU factorisation computed by DGETRF.

The results presented in figure 3 illustrate how the initial cell distribution given by (3.2) evolves under the influence of perfusion. In figure 3(a), where there is no imposed flow (static culture: $P_{u}=P_{d}=0$ ), the cell population grows and spreads symmetrically in response to the net growth rate, $k_{m}-k_{d}$, and diffusion. This is in direct contrast to the results obtained in O'Dea et al. (2008) where axially asymmetric growth was predicted in 


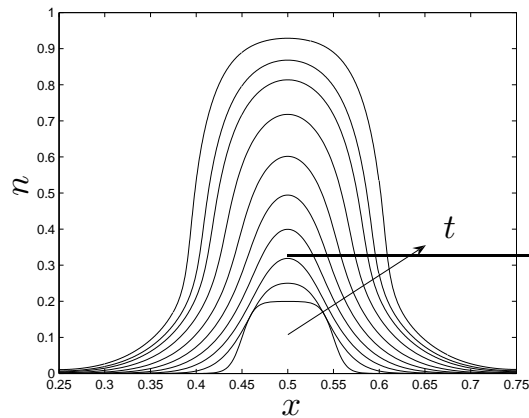

(a)

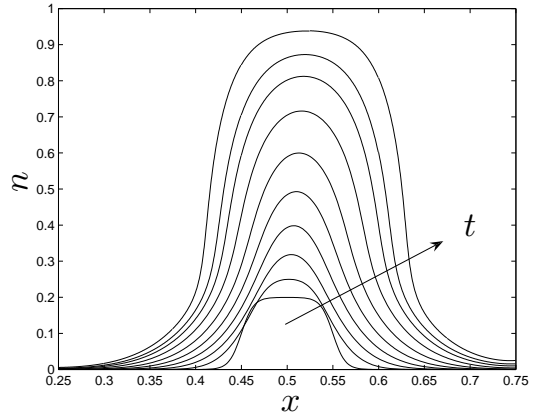

(b)

Figure 3: Evolution of the cell volume fraction $n$ for (a) static culture: $P_{u}=P_{d}=0$, (b) perfusion: $P_{u}=1, P_{d}=0.1$, at $t=0-0.297$ (in steps of $t=0.033$ ). Parameter values: $k_{m}=7.5, k_{d}=0.1, D=0.01, \theta=0.97, \nu=\chi=0.3, \delta_{a}=\delta_{b}=0.1, \mu_{n}=1.3$, $a=0.25, b=0.75$.

both static and perfusive culture (see $\S 1$ for details). Figure 3(b) illustrates the effect of perfusion on the cell phase: the tissue is advected by a small amount along the channel by the flow and an accumulation of the cell phase is observed at $x=b$. Advection may be enhanced by increasing the driving pressure gradient. The cell phase profiles in figure 3 indicate that spreading occurs before the threshold values $\hat{\mathbf{n}}=\left(\theta \nu /\left(\delta_{a}+\nu\right), \theta \chi /\left(\delta_{b}+\chi\right)\right)$ (at which $\Sigma_{n}$ and $\psi_{n s}$ change sign, corresponding to cell-cell and cell-scaffold repulsion; see (2.17) and the parameter values given in figure 3 ) due to the presence of diffusion in the model; when $n$ exceeds this value, diffusion is enhanced by repulsive forces between cells which cause the cells to spread more rapidly and produce a more uniform cell density profile at the construct centre. This phenomenon is further investigated in $\S 3.3$ by employing simplified forms for the functions $\Sigma_{n}$, and $\psi_{n s}$ to facilitate analytical progress.

Figure 4 shows the influence of the cell population on the culture medium pressure. Up- and downstream from the centrally-located dense population, equation (2.22) supplies $\partial^{2} p_{w} / \partial x^{2} \approx 0$ (since $n$ is small) so we obtain an approximately linear pressure profile; deviation from this is observed as the culture medium flows through the more denselypopulated area. Figure 4(a) shows that, initially, when the density of the cell phase is small, this deviation closely mirrors the cell phase distribution (as reported in O'Dea et al. (2008)). The pressure increase is due to the aggregative terms in the intraphase and in- 


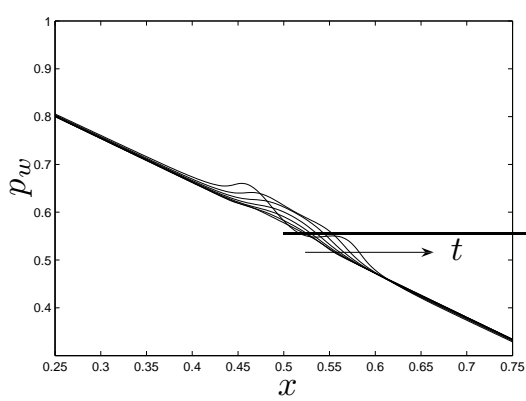

(a)

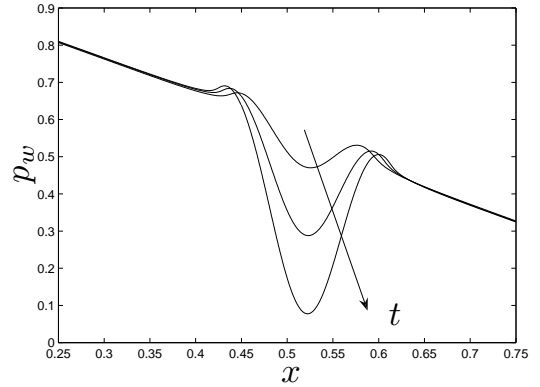

(b)

Figure 4: Evolution of the culture medium pressure for (a) early times (small $n$ ): $t=$ $0-0.231$ (in steps of $t=0.033$ ); and (b) later times (larger $n$ ): $t=0.25,0.27,0.29$, under perfusion. Parameter values as in figure 3(b).

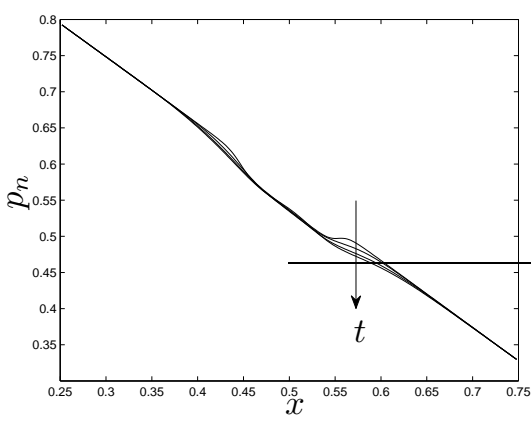

(a)

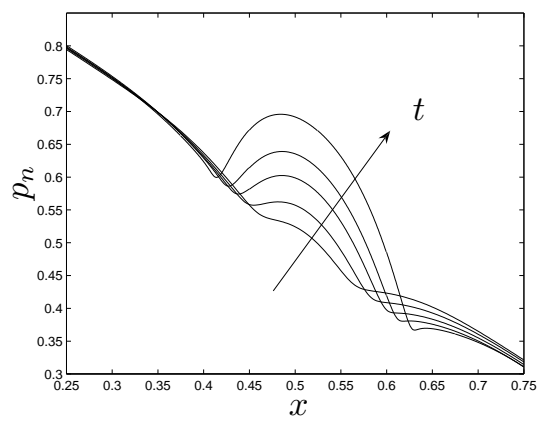

(b)

Figure 5: Evolution of the cell pressure for (a) early times ( small $n$ ): $t=0-0.13$ (in steps of $t=0.033$ ); and (b) later times (larger $n$ ): $t=0.21-0.29$ (in steps of $t=0.02$ ), under perfusion. Parameter values as in figure 3(b).

terphase pressure functions $\Sigma_{n}, \psi_{n s}$ (see equations (2.17) and (2.22)) dominating at low density. The aggregation of cells requires that culture medium is expelled from the central region and a positive culture medium pressure gradient (which drives a flow of culture medium) is therefore created at the upstream periphery of the construct (with the opposite behaviour evident downstream). As the cell phase becomes more dense, the disturbance to the culture medium pressure increases, and large deviation from the linear profile is observed in figure 4(b) due to the dominance of the repulsive terms in $\Sigma_{n}$ and $\psi_{n s}$ as $n \longrightarrow \theta$. 
As the cells repel each other, mass conservation demands that culture medium be drawn in to fill the void, corresponding to the reduction in $p_{w}$. At the periphery, where the cell population remains sparse, cell aggregation and attachment is reflected in an increase in $p_{w}$.

In figure 5 we compare the cell phase pressure for low and high cell phase density. Recall, $p_{n}$ is influenced by $p_{w}$ and intraphase and interphase interactions: $p_{n}=p_{w}+\Sigma_{n}+$ $(1-\theta) \psi_{n s}$. When $n$ is small, the behaviour is dominated by aggregation $\left(\Sigma_{n}, \psi_{n s}<0\right)$ and a small decrease in the cell phase pressure is observed. At later times (see figure 5(b)), as $n$ increases, the contribution from the repulsive terms becomes important $\left(\Sigma_{n}, \psi_{n s}>0\right)$ and a sharp increase in cell pressure is observed.

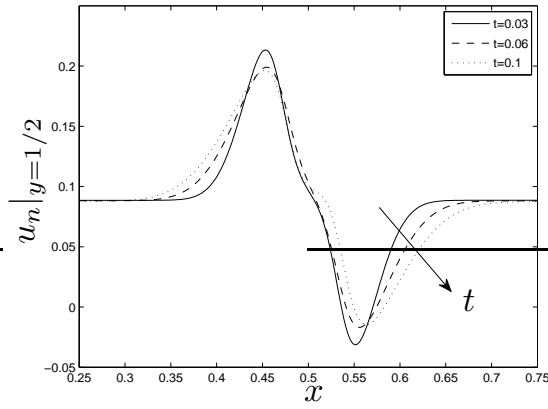

(a)

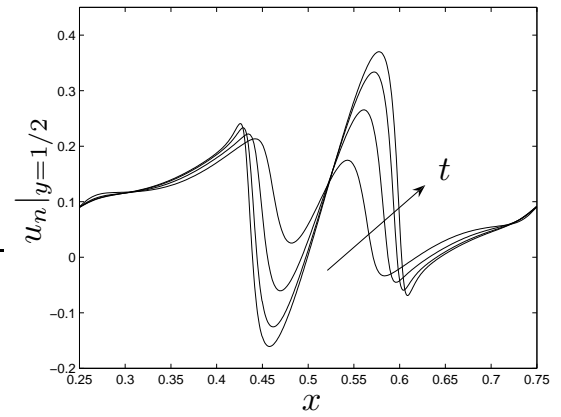

(b)

Figure 6: Evolution of the cell velocity at the channel centreline for (a) early times (small $n$ ): $t=0.033,0.066,0.1$; and (b) later times (larger $n$ ): $t=0.2,0.23,0.25,0.27$ under perfusion. Parameter values as in figure 3(b).

The velocity of the cell phase $\left(u_{n}\right)$ at the channel centreline is depicted in figure 6 . For low cell density, aggregation and attachment dominate $\left(\Sigma_{n}, \psi_{n s}<0\right)$ and we observe that cells move preferentially towards the centre to form a dense aggregate which moves downstream due to the imposed flow (figure 6(a)). As the cell volume fraction increases, repulsive effects become important $\left(\Sigma_{n}, \psi_{n s}>0\right)$ as described above. This effect is illustrated in figure 6(b) which shows that cells move outwards from the centre of the aggregate causing increased spreading at later times, as observed in figure 3. Inspection of equations (2.19)-(2.22) and (2.27)-(2.29) shows that the influence of the cell-scaffold attachment parameter $(\chi)$ is only felt through the boundary conditions; furthermore, these contributions (and those arising from cell-scaffold repulsion) scale linearly with the scaffold volume 


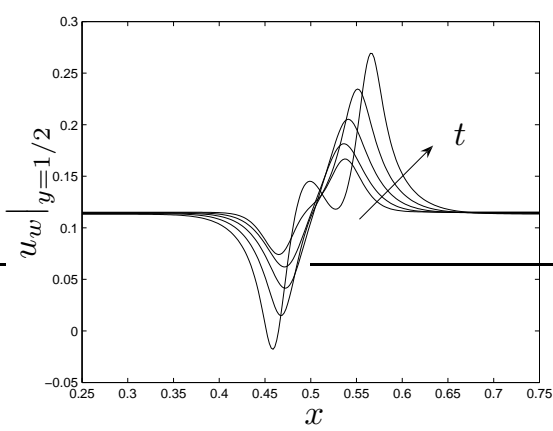

(a)

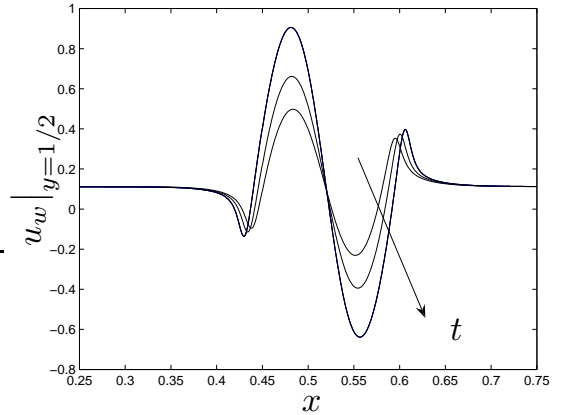

(b)

Figure 7: Evolution of the culture medium velocity at the channel centreline for (a) early times (small $n$ ): $t=0.033-0.165$ (in steps of $t=0.033$ ); and (b) later times (larger $n$ ): $t=0.25,0.27,0.29$, under perfusion. Parameter values as in figure $3(\mathrm{~b})$.

fraction which is small in these simulations. The aggregative behaviour described above is therefore dominated by cell-cell interactions.

Figure 7 shows the centreline value of the parabolic culture medium velocity profile. The flow profile remains $x$-independent prior to, and after, the densely-populated region, under the influence of the linear driving pressure gradient. For both low (figure 7(a)) and high (figure 7(b)) cell phase density, we observe that the flow speed is decreased from the upstream ambient flow velocity as the culture medium encounters the cell population; near the downstream periphery, an increase to the ambient flow is observed. At low cell density, the culture medium flow increases monotonically between the up- and downstream peripheries. As the density increases, the fluid flow between these peripheral regions changes markedly, reversing flow direction. This is due to the switch between aggregative and repulsive behaviour of the cell phase described above; to conserve mass, the culture medium velocity exhibits the opposite behaviour, being drawn into the construct's centre when cells repel each other.

Figure 8 shows the evolution of $A(t)$, which determines the culture medium pressure and its gradient in $x \leqslant a$ and $x \geqslant b$ (see equation (2.26)). The magnitude of the pressure gradient decreases with time, causing the up- and downstream flow speed to reduce; we attribute this to the increase of cell volume fraction which fills available pore space and provides increased resistance to flow.

To summarise, the results presented in this section differ significantly from those 


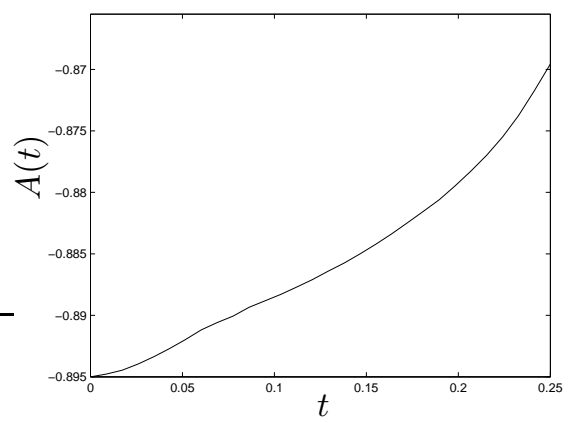

Figure 8: Evolution of the function $A(t)$. Parameter values as in figure 3(b).

predicted by the two-fluid model of O'Dea et al. (2008): consideration of cell-cell and cell-scaffold interactions, together with relaxation of the large drag assumption, results in starkly different behaviour to that found in O'Dea et al. (2008) in which axially asymmetric growth was predicted both in static and perfusive conditions ( $(1)$. Additionally, since in O'Dea et al. (2008) the limit of large viscous drag is employed, each phase moves with a common velocity and very low perfusion rates are required to prevent cells from being flushed from the scaffold. The results presented here suggest that aggregation in regions of sparse cell density acts to curtail advection, leading to movement of cells towards the centre of the aggregate; furthermore, due to mass conservation, the cell and culture medium velocities exhibit opposite behaviour. Inspection of the model equations has revealed that for $n<\theta \nu /\left(\delta_{a}+\nu\right)$ or $\theta \chi /\left(\delta_{b}+\chi\right)$, the cell behaviour is dominated by cell aggregation, with contributions from cell-scaffold attachment being small.

\subsection{Asymptotically-small cell density}

The results from the numerical scheme may be validated by considering the limit of asymptotically-small cell phase volume fraction, in which case we may construct analytic solutions to the simplified versions of (2.21) and (2.22) (with $S_{n}$ defined by (3.1)). Choosing

$$
\begin{aligned}
n(x, t) & =\delta n_{1}(x, t)+\delta^{2} n_{2}(x, t)+\cdots, \\
p_{w}(x, t) & =p_{0}(x, t)+\delta p_{1}(x, t)+\delta^{2} p_{2}(x, t)+\cdots,
\end{aligned}
$$


where $0<\delta \ll 1$, we obtain the following system of linear PDEs:

$$
\begin{aligned}
& \frac{\partial n_{1}}{\partial t}+\gamma \frac{\partial n_{1}}{\partial x}=\left(k_{m}-k_{d}\right) n_{1}+D \frac{\partial^{2} n_{1}}{\partial x^{2}}, \\
& \frac{\partial^{2} p_{0}}{\partial x^{2}}=0, \quad \frac{\partial^{2} p_{1}}{\partial x^{2}}=-\beta \frac{\partial n_{1}}{\partial x},
\end{aligned}
$$

where $\gamma$ and $\beta$ are defined as follows:

$$
\gamma=-\frac{1}{12 \mu_{n}} \frac{\partial p_{0}}{\partial x}, \quad \beta=\frac{\bar{\mu}}{\theta} \frac{\partial p_{0}}{\partial x} .
$$

Considering (2.27)-(2.29) and employing the additional expansion

$$
A(t)=A_{0}(t)+\delta A_{1}(t)+\cdots,
$$

it may be shown that appropriate boundary conditions are:

at $\mathcal{O}(1)$

$$
\left.\frac{\partial p_{0}}{\partial x}\right|_{x=a, b}=\frac{A_{0}}{\theta},\left.\quad p_{0}\right|_{x=a}=\frac{A_{0} a+P_{u}}{\theta},\left.\quad p_{0}\right|_{x=b}=\frac{A_{0}(b-1)+P_{d}}{\theta} ;
$$

at $\mathcal{O}(\delta)$ :

$$
\begin{gathered}
\left.\frac{\partial n_{1}}{\partial x}\right|_{x=a, b} ^{=}=-\frac{A_{0} n_{1}}{12 \theta \mu_{n} D},\left.\quad \frac{\partial p_{1}}{\partial x}\right|_{x=a, b}=\frac{A_{1}}{\theta}+\frac{A_{0} n_{1}}{\theta^{2}}+\frac{12 D}{\theta} \frac{\partial n_{1}}{\partial x},(3.10 a, b) \\
\left.p_{1}\right|_{x=a}=\frac{A_{1} a+(1-\theta) \chi n_{1}}{\theta},\left.\quad p_{1}\right|_{x=b}=\frac{A_{1}(b-1)+(1-\theta) \chi n_{1}}{\theta} .(3.11 a, b)
\end{gathered}
$$

We therefore have four conditions on each of the pressures $p_{0}, p_{1}$; two of which are imposed as boundary conditions, the remaining equations being used to calculate $A_{0}$ and $A_{1}$. As previously, the overspecification of the functions $A_{0}, A_{1}$ results from the imposition of continuity of total flux. When satisfied, the additional conditions guarantee continuity of flux.

For simplicity, we consider the solution of equations (3.5) and (3.6) in the limit $D=0$, for which the interface between the cell phase and the surrounding culture medium is sharp. The cell population is then confined within two moving boundaries, $x=l(t), r(t)$, within the scaffold region $a \leqslant x \leqslant b$.

It is trivial to show that $A_{0}=P_{d}-P_{u}$ and the leading-order pressure, $p_{0}$, is given by

$$
p_{0}(x, t)=\frac{\left(P_{d}-P_{u}\right) x+P_{u}}{\theta} .
$$


We may now proceed with the solution of equation (3.5) with $D=0$ since the constant, $\gamma$, is given by equation (3.7a). We first specify an appropriate initial cell phase distribution as follows:

$$
n_{1}(x, 0)= \begin{cases}\bar{n}(x) & l(0) \leqslant x \leqslant r(0), \\ 0 & \text { otherwise }\end{cases}
$$

wherein $\bar{n}(x)$ is an as yet unspecified function and $x=l(0), r(0)$ are the initial positions of the interfaces $l(t), r(t) ;(3.10 a)$ is redundant since $n_{1}=0$ for $a \leqslant x<l, r<x \leqslant b$. The solution, $n_{1}(x, t)$ takes the form of a travelling-wave:

$$
n_{1}(x, t)= \begin{cases}\bar{n}(x-\gamma t) e^{\left(k_{m}-k_{d}\right) t} & l(t) \leqslant x \leqslant r(t), \\ 0 & \text { otherwise }\end{cases}
$$

where $l(t)=l(0)+\gamma t, r(t)=r(0)+\gamma t$, representing exponential growth of a cell population at a rate $k_{m}-k_{d}$ which is advected along the channel at speed $\gamma$. This behaviour is valid for the very early stages of cell growth during which behaviour is dominated by uniform proliferation and cell spreading is negligible.

The correction to the culture medium pressure $p_{1}$ is given by equation (3.6b), and, in addition to the conditions (3.9)-(3.11) at $x=a, b$, must obey the following jump conditions across $x=l(t), r(t)$ :

$$
\left[\theta p_{1}\right]_{-}^{+}=\left[\chi(1-\theta) n_{1}\right]^{+}, \quad\left[\theta \frac{\partial p_{1}}{\partial x}\right]_{-}^{+}=\left[\frac{\left(P_{u}-P_{d}\right) \bar{\mu} n_{1}}{\theta}\right]^{+}
$$

where $[. .]^{+}$and $[. .]^{-}$denote the limiting values from the cell/culture medium/scaffold region $(l(t) \leqslant x \leqslant r(t))$ and the culture medium/scaffold regions $(a \leqslant x<l(t), r(t)<$ $x \leqslant b)$, respectively and $[. .]_{-}^{+}$denotes the jump across either interface.

To determine the correction to the pressure in the culture medium, we must specify the initial cell phase distribution, $\bar{n}(x)$. For simplicity we choose $\bar{n}(x)=\hat{n}$, where $\hat{n}$ is constant. We obtain:

$$
p_{1}(x, t)= \begin{cases}\widetilde{P} e^{\bar{k} t}[l(t)-r(t)] x & a \leqslant x<l(t), \\ \widetilde{P} e^{\bar{k} t}[1+l(t)-r(t)] x+e^{\bar{k} t}(\bar{\chi}-\widetilde{P} l(t)) & l(t) \leqslant x \leqslant r(t), \\ \widetilde{P} e^{\bar{k} t}[l(t)-r(t)](x-1) & r(t)<x \leqslant b,\end{cases}
$$

where $\bar{k}=k_{m}-k_{d}, \bar{\chi}=\chi(1-\theta) \hat{n} / \theta, \bar{\mu}=1 / \mu_{n}-1$ and $\widetilde{P}=\left(P_{u}-P_{d}\right) \bar{\mu} \hat{n} / \theta^{2}$.

The evolution of the cell volume fraction, $n_{1}$, is shown in figure 9 . The corresponding pressure correction, $p_{1}$, and the culture medium pressure (to $\mathcal{O}(\delta)$ accuracy) $p_{w}=p_{0}+$ $\delta p_{1}$, are shown in figure 10. The correction to the pressure $\left(p_{1}\right)$ is an order of magnitude smaller than the leading-order pressure $\left(p_{0}\right)$; in order that the effects are visible in figure 
10 (b), the small parameter is chosen to be $\delta=1$. With the exception of the diffusion coefficient, $D$, the parameter values are chosen to be the same as those used in $\S 3.1$.

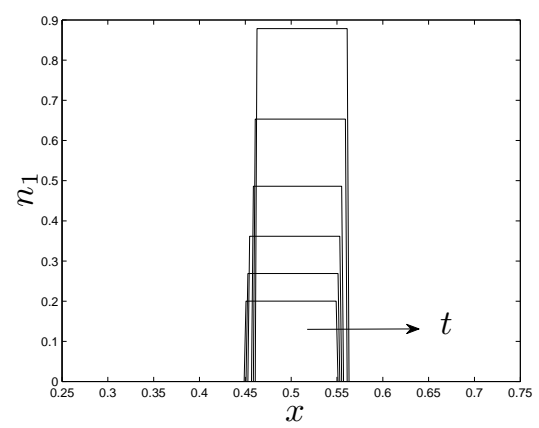

Figure 9: Evolution of the cell volume fraction, $n_{1}$, under perfusion at $t=0-0.2$ (in steps of $t=0.04)$. $D=0, \delta=1$, other parameter values as in $\S 3.1$.

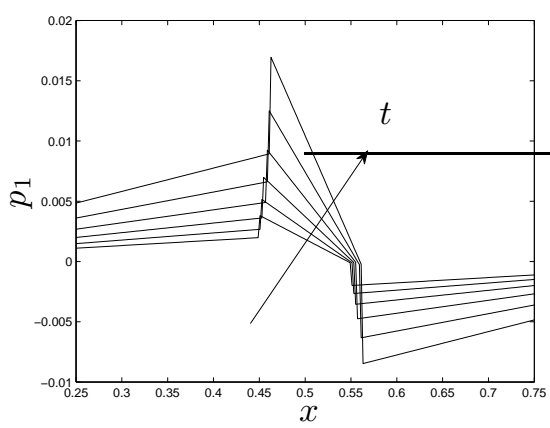

(a)

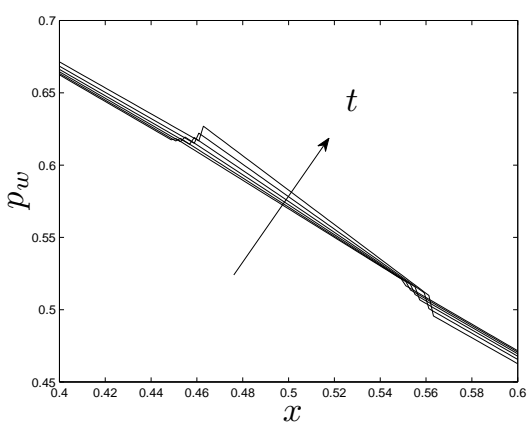

(b)

Figure 10: Evolution of (a) the pressure correction, $p_{1}$ and, (b) the culture medium pressure, $p_{w}=p_{0}+\delta p_{1}$ in a magnified region within $a \leqslant x \leqslant b$, under perfusion at $t=0-0.2$ (in steps of $t=0.04$ ). Parameters as in figure 9 .

As noted above, the solution in the sharp interface limit predicts that the cell population grows exponentially with growth rate $k_{m}-k_{d}$, while being advected along the channel at speed $\gamma$; the width of the population remains unchanged. For validation purposes, the corresponding advection speed predicted by the numerical scheme developed in $\$ 3.1$ may be readily calculated by tracking the position of the maximum value of $n$. Figure 11(a) shows how the position of this numerically-calculated maximum value compares to the position 


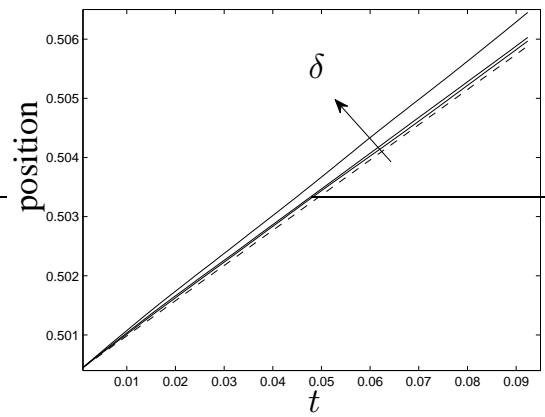

(a)

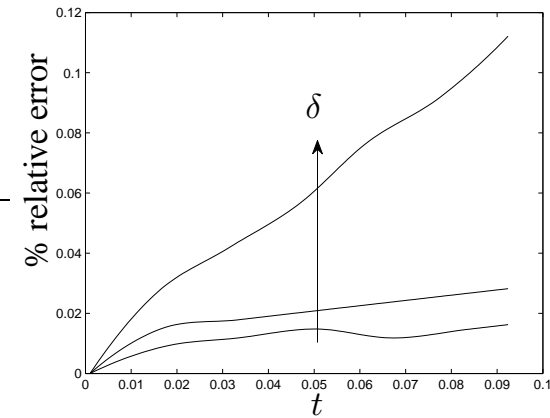

(b)

Figure 11: (a) Comparison of the numerically-computed position of the maximum value of $n(-)$ compared to the predicted position of the travelling wave (- -) and, (b) the \% relative error between the calculated and predicted position for $\delta=1 / 25,1 / 5,1$. The arrows indicate the direction of increasing $\delta$.

predicted by the travelling-wave solution (3.14), and figure 11(b) depicts the \% relative error between the numerically-calculated and analytically-predicted positions over time for different values of the small parameter $\delta$. As $\delta$ is decreased, the numerical prediction for the advection speed approaches $\gamma$, and the $\%$ relative error decreases (for $\delta=1 / 25$, the $\%$ error is $\left.\mathcal{O}\left(10^{-2}\right)\right)$.

The perturbation $\left(p_{1}\right)$ to the culture medium pressure is found to be piecewise linear, with positive gradient in the up- and downstream regions where $n_{1}=0$ and negative gradient where cells are present $(l(t) \leqslant x \leqslant r(t))$. Upstream, the sharp interface limit predicts a small increase to the leading-order pressure; downstream, a small decrease is observed. Comparison of the predicted pressure shown by figure 10(b) and the culture medium pressure calculated in $\S 3.1$ (figure 4(a)) shows qualitative agreement. Furthermore, considering the boundary conditions (2.27a) and (2.28a) and the behaviour of $A(t)$ (see figure 8 which indicates that $A(t)<0$ and that $|A(t)|$ decreases with time), we see that at $x=a$ the culture medium pressure increases over time; at $x=b$, the pressure decreases. This behaviour is evident from figure 10(b), indicating that the culture medium pressure $\left(p_{w}=p_{0}+\delta p_{1}\right)$ predicted in this asymptotic limit reproduces that of the system (2.21), (2.22), (2.27)-(2.29) when the cell density is $\mathcal{O}(1)$.

It is possible to obtain better agreement between the analytical and numerical solutions by relaxing the sharp interface assumption so that $D>0$. In appendix A we construct 
analytic solutions using Greens functions for the case $D>0$.

\subsection{Analysis of a simplified model of cell-cell and cell-scaffold inter- action}

To investigate further the effect of intraphase pressure and interphase traction on cell behaviour, especially the switch between aggregative and repulsive behaviour observed in the numerical simulations (see figures 4-7), we now simplify the intraphase pressure and interphase traction functions defined by equations (2.17), replacing them with the following piecewise-constant forms:

$$
\Sigma_{n}(n)=\left\{\begin{array}{cc}
-\nu & n<N_{\Sigma}, \\
\delta_{a} & n \geqslant N_{\Sigma},
\end{array} \quad \psi_{n s}(n)=\left\{\begin{array}{rl}
-\chi & n<N_{\psi}, \\
\delta_{b} & n \geqslant N_{\psi},
\end{array}\right.\right.
$$

where $N_{\Sigma}, N_{\psi}$ are the threshold values at which repulsive forces between cells dominate those associated with aggregation and at which the cells become repelled from the scaffold, respectively. For simplicity, in the following we set $N_{\Sigma}=N_{\psi}=N$ and we further assume that the viscosities of the culture medium and cell phases are equal $\left(\mu_{n}=1\right)$. Under these simplifications, equation (2.22) reduces to:

$$
\theta \frac{\partial^{2} p_{w}}{\partial x^{2}}=\left\{\begin{aligned}
\nu \frac{\partial^{2} n}{\partial x^{2}} & n<N \\
-\delta_{a} \frac{\partial^{2} n}{\partial x^{2}} & n \geqslant N
\end{aligned}\right.
$$

Assuming $n<N$ at $x=a, b$, the corresponding boundary conditions (2.27)-(2.29) are

$$
\begin{aligned}
& \left.p_{w}\right|_{x=a}=\frac{A a+P_{u}+\alpha n}{\theta},\left.\quad \frac{\partial p_{w}}{\partial x}\right|_{x=a, b}=\frac{A+12 D \frac{\partial n}{\partial x}}{\theta-n} \\
& \left.p_{w}\right|_{x=b}=\frac{A(b-1)+P_{d}+\alpha n}{\theta},\left.\frac{\partial n}{\partial x}\right|_{x=a, b}=\frac{A n}{(\theta-n)(\nu-12 D)-12 D n}
\end{aligned}
$$

where $\alpha=\nu+(1-\theta) \chi$.

We proceed by considering separately the regions in which $n<N$ and $n \geqslant N$, assuming that $n, \partial n / \partial x, p_{w}$ and $\partial p_{w} / \partial x$ are continuous at $n=N$. Equations (3.18)-(3.20) yield expressions for the culture medium pressure in each region, substitution of which into (2.21) yields nonlinear advection-diffusion equations for the cell volume fraction (omitted), in which the effective diffusion coefficients are defined:

$$
D=D-\frac{(\theta-n) \nu}{12 \theta}, n<N ; \quad D(n)=D+\frac{(\theta-n) \delta_{a}}{12 \theta}, n \geqslant N .
$$

This system requires non-trivial numerical solution, offering little benefit over numerical solution of the original equations. However, this analysis does provide some insight 
into the behaviour of the cells: the modified diffusion coefficients, indicate that when aggregation dominates $(n<N)$ ), the diffusive transport of the cells is reduced; conversely, when $n \geqslant N$, repulsive effects dominate and the cellular diffusion coefficients are increased.

Aggregation-enhanced cell behaviour is most clearly illustrated by considering the cell phase velocity defined by $(2.19 b)$. In view of the simplified forms (3.17), we find that the cell velocity at the channel centreline for $n<N$ is given by

$$
u_{n} \approx-\frac{1}{8 \mu_{n}}\left(\frac{\partial p_{w}}{\partial x}-\frac{\nu}{n} \frac{\partial n}{\partial x}\right),
$$

so that for small $n$, the second term dominates and cells tend to move up gradients of cell density.

\subsection{Summary}

In this section, we have considered the uniform growth of a tissue construct. We presented numerical simulations which indicate that the consideration of interactions between adjacent cells and between cells and the scaffold leads to distinctly different cell behaviour as the construct density increases: cell aggregation and attachment being replaced by repulsion. The accuracy of the numerical simulations was established by constructing analytic solutions in the limit of asymptotically-small cell density. To further investigate the behavioural switch observed in the numerical simulations, we employed simplified functional forms for the cell-cell and cell-scaffold interactions. Our analysis indicated that the cells' diffusive behaviour is reduced or augmented depending upon the relative importance of cell aggregation and repulsion.

\section{Mechanotransduction}

We now include a simple mechanotransduction mechanism which regulates the cells' proliferative response. By extending our model to consider the effect of coupling the growth of the cell population to the local environment, we can determine the characteristic growth pattern associated with specific mechanical stimuli; in tandem with experimental data, this will allow optimisation of culture conditions and could provide an indication of which stimuli regulate cell proliferation. We couple the growth of the cell population to the following stimuli: contact inhibition caused by cell-cell interactions, the effect of stress caused by increases in local cell density and the influence of the external fluid dynamics. 
The relevance of our modelling framework hinges on the appropriate choice of $S_{n}$; we pause here to highlight an important restriction on its form. Equation (2.21) is derived by averaging the conservation of mass equation for the culture medium phase (in the transverse direction), implying $S_{n}=S_{n}(x, t)$; consequently, explicit coupling between the shear stress induced by the flow of culture medium (which is dependent on $y$ ) and the cell growth response is prohibited. The gross effect of this coupling may still be incorporated by noting that the averaged flow-induced shear stress experienced by the cells is proportional to the culture medium velocity. In view of equation $(2.19 a)$, we therefore model the shear stress as being proportional to the gradient of the culture medium pressure. In the following, we consider in turn the following choices: $S_{n}(n), S_{n}\left(n, p_{n}\right), S_{n}\left(n,\left|\partial p_{w} / \partial x\right|\right)$.

\subsection{Cell density dependence: $S_{n}=S_{n}(n)$}

We identify three distinct stages in the behaviour of the cell population: (i) a proliferative stage, $S_{n}=k_{1 n} n$; (ii) an ECM-producing stage, $S_{n}=k_{2 n} n$; and (iii) an apoptotic stage, $S_{n}=-k_{d} n$. These represent the effects of contact inhibition and residual stresses caused by growth on the phenotypic progression of cells. Contact inhibition and high stress levels inhibit cell division, whilst a moderate level of stress appears to enhance tissue growth (Chaplain et al., 2006; Roose et al., 2003). We therefore choose $k_{2 n}>k_{1 n}$ so that the rate of cell phase growth is increased during the ECM-production phase; we remark here that since the cell phase comprises cells and ECM, it is not possible to distinguish between cell proliferation and ECM deposition or cell death and ECM degradation in this model. For simplicity, we assume that the rates of growth and death $\left(k_{1 n}, k_{2 n}, k_{d}\right)$ are constant. The threshold cell densities that separate these three types of behaviour are denoted $n_{1}^{\prime}$ and $n_{2}^{\prime}$.

We employ step functions to represent this behaviour; the net rate of growth and death of the cell phase, denoted $\kappa(n)$, is illustrated by figure 12 and is related to $S_{n}$ as follows:

$$
S_{n}(n)=\left[k_{1 n} \mathrm{H}\left(n_{1}^{\prime}-n\right)+k_{2 n} \mathrm{H}\left(n-n_{1}^{\prime}\right)-\left(k_{2 n}+k_{d}\right) \mathrm{H}\left(n-n_{2}^{\prime}\right)\right] n=\kappa(n) n,
$$

where $H(n)$ is the Heaviside step function and without loss of generality, we specify $\kappa(n)=k_{2 n}$ at the threshold values $n=n_{1}^{\prime}, n_{2}^{\prime}$. Step functions for density- and nutrientdependent growth have been employed by Byrne \& Preziosi (2003a) in which the switch between two density-dependent responses was modelled; a (mollified) piecewise constant response was employed by Chaplain et al. (2006). Here, we consider three distinct growth phases in each of which the proliferative rate is constant.

Figure 13(a) shows the effect of this modified mass transfer term on the evolution of the cell phase when subjected to perfusion, and the regions in which the different growth 


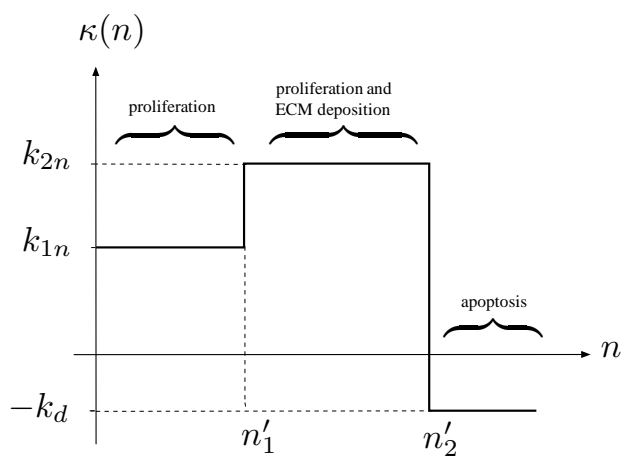

Figure 12: Schematic representation of the progression of the cells from a proliferative phase to an apoptotic phase, via an ECM-producing phase in response to the local cell density.

responses occur. The corresponding culture medium and cell phase pressures are shown in figures 13(b) and 14. The velocity of each phase at the channel centreline is shown in figure 15; for clarity, only the velocities arising at later times, once $n$ has reached the threshold value $n=n_{2}^{\prime}$ at some point in the domain, are shown.

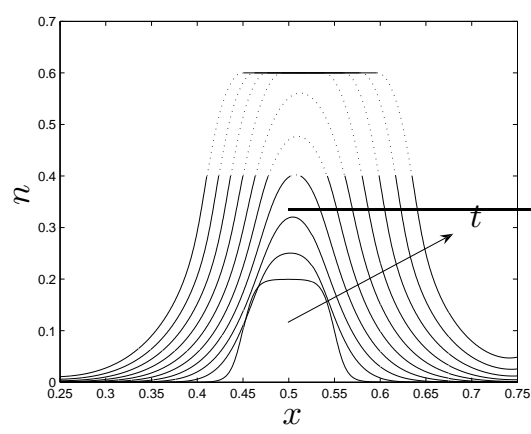

(a)

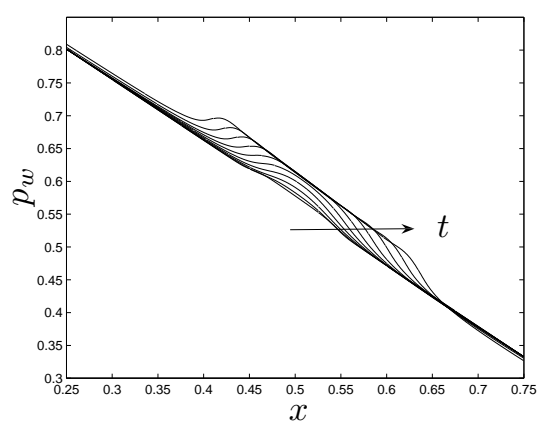

(b)

Figure 13: The evolution of (a) the cell volume fraction, $n<n_{1}^{\prime}$ and $n>n_{2}^{\prime},(-) ; n_{1}^{\prime} \leqslant$ $n \leqslant n_{2}^{\prime},(\cdots)$ and, (b) the pressure of the culture medium, at $t=0-0.35$ (in steps of $t \approx 0.038$ ) for growth behaviour defined by (4.1) and perfusive culture: $P_{u}=1, P_{d}=0.1$, $k_{1 n}=6.5, k_{2 n}=7.5, k_{d}=1, D=0.01, \theta=0.97, n_{1}^{\prime}=0.4, n_{2}^{\prime}=0.6$.

Inspection of figure 13(a) reveals that the growth of the cell phase ceases when $n=n_{2}^{\prime}$, due to the progression from a proliferative to an apoptotic phase. Despite the presence of apoptosis in our model, re-entry into the proliferative phase ensures that, once attained, the 


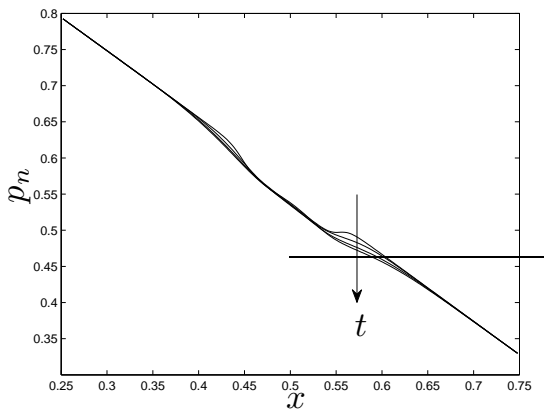

(a)

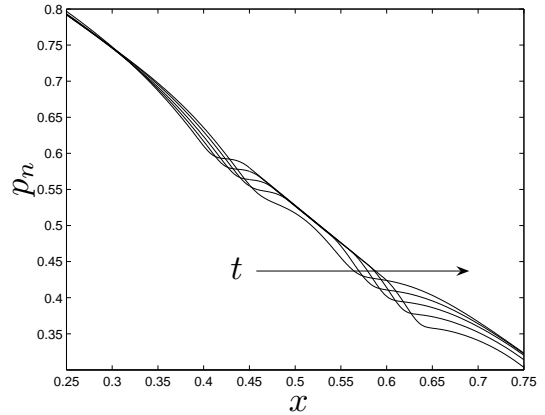

(b)

Figure 14: The evolution of the pressure of the cell phase for (a) early times (small $n$ : $t=0-0.15$, in steps of $t=0.0375)$ and, (b) later times (larger $n: t=0.2-0.35$ in steps of $t=0.05$ ), for growth behaviour defined by (4.1) and perfusive culture. Parameter values as per figure 13 .

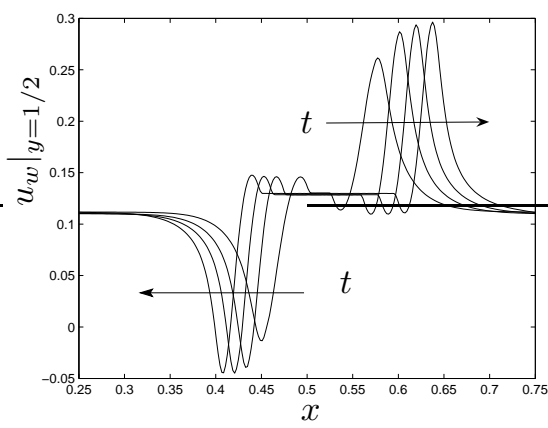

(a)

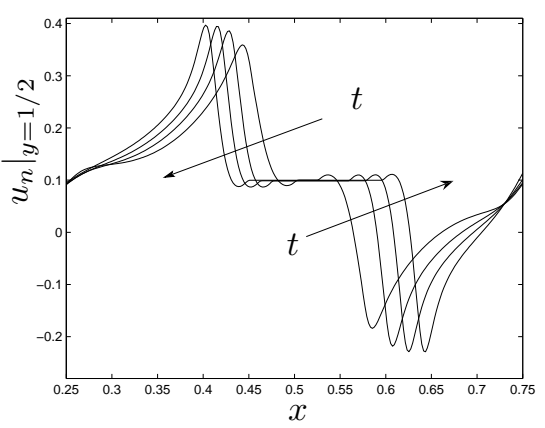

(b)

Figure 15: The evolution of (a) the velocity profile of the culture medium, (b) the velocity profile of the cell phase (at the channel centreline), at $t=0.2-0.35$ (in steps of $t=0.05$ ) for growth behaviour defined by (4.1) and perfusive culture. Parameter values as per figure 13.

density of the cell phase does not fall below $n=n_{2}^{\prime}$. Figures 13(b), 14 and 15 indicate that the pressure and velocity of each phase exhibit similar behaviour to those shown in figures 4(a), 5(a), 6(a) and 7(a). Since excessive cell proliferation is prevented, the repulsive terms in the intraphase pressure and interphase traction functions are unable to dominate and the cells aggregate to a form a dense population, which is advected under the imposed 
flow. Some repulsion is evident in figure 14(b); however, the behaviour shown in 5(b) is prevented by curtailed cell phase growth. Similarly, the dramatic flow reversal observed in figures 6(b) and 7(b) does not occur (limited upstream flow of culture medium due to cell aggregation is observed at the upstream periphery of the construct, as in figure 7(a)); rather, the flow attains a constant value in the region where $n=n_{2}^{\prime}$.

\subsection{Cell density and pressure dependence: $S_{n}=S_{n}\left(n, p_{n}\right)$}

An alternative way to model the tendency of cells to adapt their behaviour in response to their local density is to consider the pressure of the cell phase as an indicator of cell density; i.e. $S_{n}\left(n, p_{n}\right)$. Since $p_{n}$ is intimately connected to the pressure of the culture medium, this choice has the added advantage of including the response of cells to the local fluid dynamics.

We model the cells' pressure-dependent response in a similar manner to that outlined above and assume that at intermediate pressure, the cells exhibit enhanced proliferation and ECM deposition; at low pressure, the cells enter a state of relative quiescence in which proliferation and ECM deposition are greatly reduced; at high pressure, the cells become apoptotic. This behaviour is consistent with Roelofsen et al. (1995) in which it was reported that excessive hydrostatic pressure $(>200 \mathrm{kPa})$ has an inhibitory effect on bone-specific gene expression in murine osteoblast-like cells. Introducing threshold cell pressures at which the cell proliferation is heightened $\left(p_{n 1}^{\prime}\right)$ and apoptosis is stimulated $\left(p_{n 2}^{\prime}\right)$, we represent the mass transfer term with step functions, as defined below and illustrated by figure 16(a):

$S_{n}\left(n, p_{n}\right)=\left[k_{1 p} \mathrm{H}\left(p_{n 1}^{\prime}-p_{n}\right)+k_{2 p} \mathrm{H}\left(p_{n}-p_{n 1}^{\prime}\right)-\left(k_{2 p}+k_{d}\right) \mathrm{H}\left(p_{n}-p_{n 2}^{\prime}\right)\right] n=\kappa\left(p_{n}\right) n$.

Within our numerical scheme we choose $\kappa\left(p_{n}\right)=k_{2 p}$ at $p_{n}=p_{n 1}^{\prime}, p_{n 2}^{\prime}$.

Comparison of figures 13(a) and 16(b) demonstrates the effect of $S_{n}\left(n, p_{n}\right)$ on the growth of the cell phase: rather than being arrested at a threshold density, the growth of the cell phase is skewed towards the downstream boundary $x=b$. This is due to the interplay between the imposed pressure, $p_{w}$, (which dominates $p_{n}$ when $n$ is small) and the repulsive intraphase pressure and interphase traction contributions (which cause a dramatic increase in $p_{n}$ when $n$ becomes larger; see equation (2.17)). Growth of the cell phase near $x=a$ is inhibited because the culture medium pressure is high there $\left(\kappa\left(p_{n}\right)=-k_{d}\right)$; near $x=b$, growth is reduced $\left(\kappa\left(p_{n}\right)=k_{1 p}<k_{2 p}\right)$; and between these two regions, enhanced growth is initially observed until the cell pressure achieves the threshold $p_{n 2}^{\prime}$ (see the last 


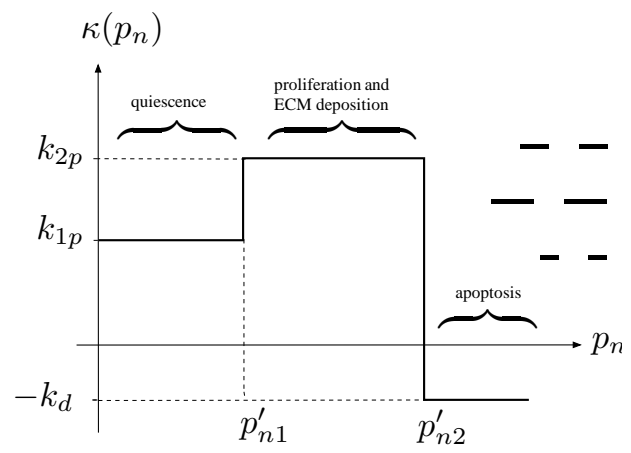

(a)

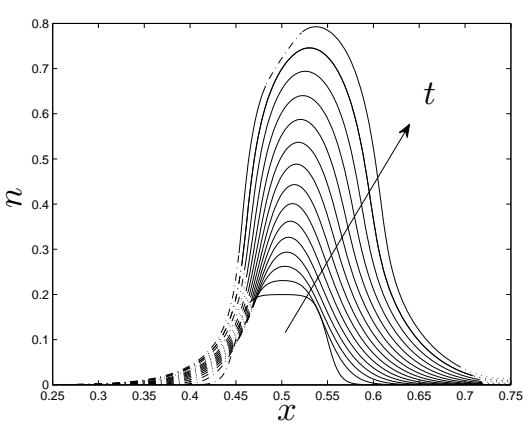

(b)

Figure 16: (a) Schematic representation of the progression of the cells from a quiescent phase to an apoptotic phase, via a proliferative phase in response to the pressure of the cell phase, $p_{n}$; (b) the evolution of the cell volume fraction at $t=0-0.28$ (in steps of $t=0.02), p_{n}>p_{n 2}^{\prime},(-.-) ; p_{n 1}^{\prime} \leqslant p_{n} \leqslant p_{n 2}^{\prime},(-) ; p_{n}<p_{n 1}^{\prime},(\cdots)$, for growth behaviour defined by (4.2) and perfusive culture: $P_{u}=1, P_{d}=0.1, k_{1 p}=4, k_{2 p}=7.5, k_{d}=2$, $D=0.01, \theta=0.97, p_{n 1}^{\prime}=0.35, p_{n 2}^{\prime}=0.6$.

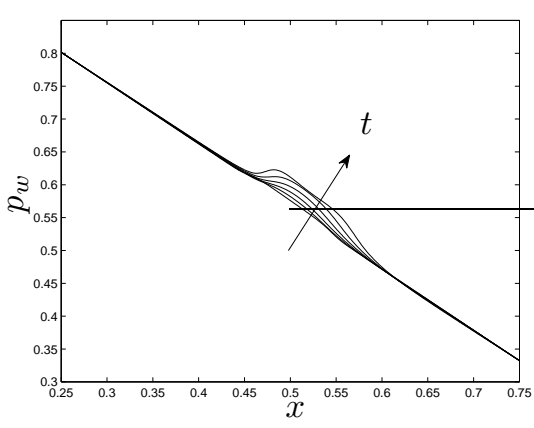

(a)

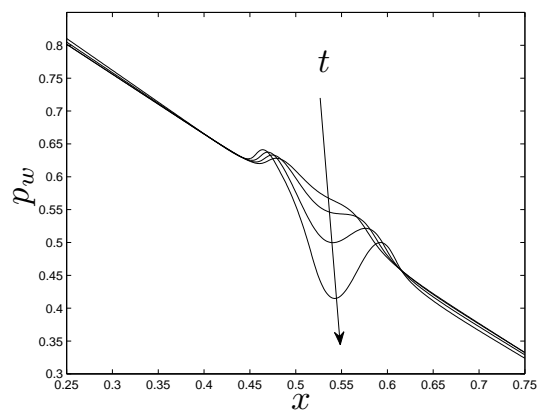

(b)

Figure 17: The evolution of the pressure of the culture medium for (a) early times (small $n$ ): $t=0-0.2$ (in steps of $t=0.04$ ), (b) later times (larger $n$ ): $t=0.22-0.28$ (in steps of $t=0.02$ ), for growth behaviour defined by (4.2) and perfusive culture. Parameter values as per figure 16 .

line in figure 16). Comparison between figures 5 and 18 shows that the cell pressure is not dramatically affected by this changed cell distribution until the upper threshold $p_{n 2}^{\prime}$ is reached within the densely-populated region. Here, transition to the apoptotic phase 


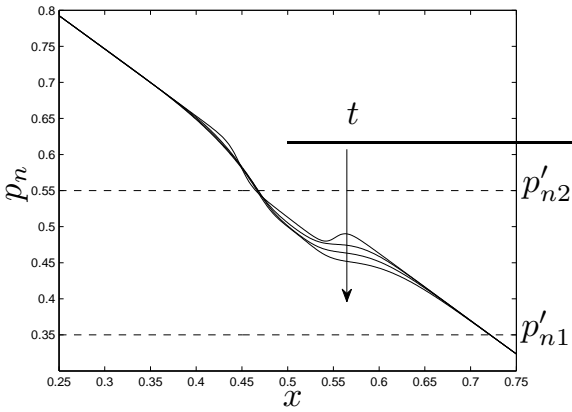

(a)

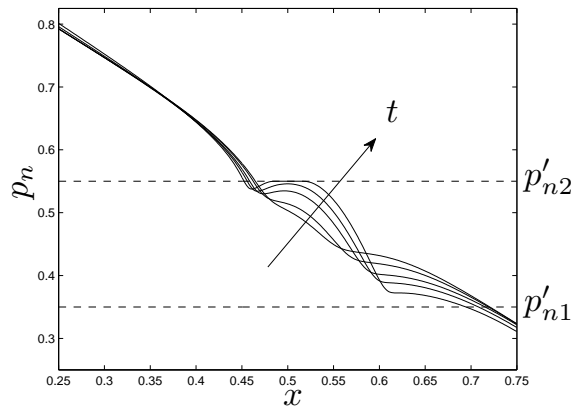

(b)

Figure 18: The evolution of the pressure of the cell phase for (a) early times ( $\operatorname{small} n$ ): $t=0-0.12$ (in steps of $t=0.4$ ), (b) later times (larger $n$ ): $t=0.16-0.28$ (in steps of $t=0.03$ ), for growth behaviour defined by (4.2) and perfusive culture. Parameter values as per figure 16 .

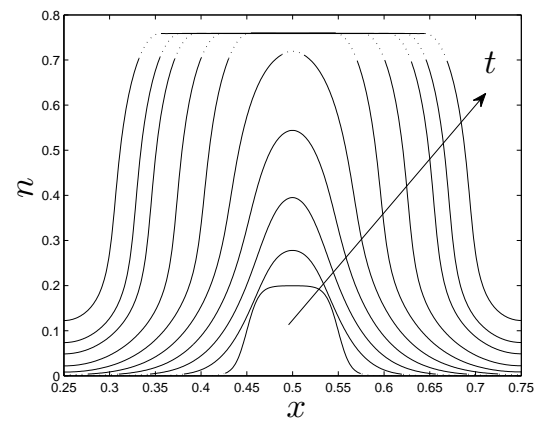

Figure 19: The evolution of the cell volume fraction at $t=0-0.3$ (in steps of $t=0.033$ ), $p_{n}<p_{n 1}^{\prime}, p_{n}>p_{n 2}^{\prime},(-) ; p_{n 1}^{\prime} \leqslant p_{n} \leqslant p_{n 2}^{\prime},(\cdots)$, for growth behaviour defined by (4.2) and static culture: $P_{u}=0=P_{d}, k_{1 p}=7.5, k_{2 p}=9, p_{n 1}=0, p_{n 2}^{\prime}=0.01$, other parameters as in figure 16 .

prevents $p_{n}$ from exceeding $p_{n 2}^{\prime}$ (see the last line in figure 18(b)). Similarly, figures 4 and 17 show that the culture medium pressure is qualitatively similar to that found previously. For brevity, the velocities of each phase are not given here since (except at late times) they will be qualitatively similar to those found in $\S 3.1$.

Figure 19 shows the predicted construct morphology obtained in static culture conditions $\left(P_{u}=P_{d}=0\right)$, indicating that in the absence of perfusion, pressure-regulated 
growth results in a construct whose composition is qualitatively similar to that resulting from density-regulated growth. Comparison with figure 13(a), which depicts the construct morphology resulting from density-regulated growth under perfusion, shows that the constructs may be distinguished by the asymmetry introduced by the flow. Qualitatively indistinguishable constructs are obtained in static conditions (results omitted).

Using a two phase model, O'Dea et al. (2008) have also demonstrated that in the absence of perfusion, cell density and pressure-mediated growth result in indistinguishable constructs; the similarity of the constructs produced was a consequence of the simplified model in which the pressure was directly proportional to the cell distribution. In this three phase model, where the relationship between the cell phase distribution and its pressure is more complex, the net result is the same; however, the mechanism is different. In static culture, dominance of the aggregation and scaffold affinity parameters at low cell density ensures that $p_{n}<0$ and tissue growth is determined by the reduced growth rate, $\kappa\left(p_{n}\right)=k_{1 p}$; as the density increases, the repulsive terms become important, causing an increase in cell phase pressure until $p_{n}$ achieves the upper threshold and the cells become apoptotic, preventing the cell density from further increase. Cells near the periphery of the aggregate (where the density and associated cell pressure are lower) proliferate at a rate $k_{1 p}$ or $k_{2 p}$ depending upon the value of $p_{n}$ (cells proliferating at $\kappa\left(p_{n}\right)=k_{2 p}$ are indicated by the dotted line in figure 19). Eventually, these cells achieve sufficiently high density to cause the pressure to attain the upper threshold, resulting in curtailed growth. In this way, a construct whose density is approximately uniform is attained. We note that these results were obtained for the case $P_{u}=P_{d}=0$; similar behaviour is obtained for $P_{u}=P_{d}>0$ depending upon the choice of thresholds $p_{n 1}^{\prime}, p_{n 2}^{\prime}$.

\subsection{Shear stress dependence: $S_{n}=S_{n}\left(n,\left|\partial p_{w} / \partial x\right|\right)$}

We now consider the effect of coupling the growth of the cell phase to the shear stress induced by the external fluid dynamics; i.e. $S_{n}\left(n,\left|\partial p_{w} / \partial x\right|\right)$. We employ the same modelling techniques as previously, and assume that at an intermediate level of shear stress, the rates of proliferation and ECM deposition are heightened; for low shear stress, the proliferation and ECM deposition rates are reduced; and for excessively high shear stresses the cells become damaged and enter apoptosis. In this case, however, we find that to ensure a smooth numerical solution, we must employ a smoothed version of the functional form for 
the mass transfer term, $S_{n}\left(n,\left|\partial p_{w} / \partial x\right|\right)$ defined as follows, and depicted in figure 20(a):

$$
\begin{aligned}
S_{n}\left(n,\left|\frac{\partial p_{w}}{\partial x}\right|\right) & =\left\{-\frac{k_{1 \tau}-k_{2 \tau}}{2}\left(\tanh \left[g\left(\left|\frac{\partial p_{w}}{\partial x}\right|-P_{1}^{\prime}\right)\right]-1\right)\right. \\
& \left.\quad-\frac{k_{2 \tau}+k_{d}}{2}\left(\tanh \left[g\left(\left|\frac{\partial p_{w}}{\partial x}\right|-P_{2}^{\prime}\right)\right]-1\right)-k_{d}\right\} n \\
= & \kappa\left(\left|\frac{\partial p_{w}}{\partial x}\right|\right) n
\end{aligned}
$$

In (4.3), the threshold values at which the rate of cell proliferation and ECM deposition are heightened and the necrotic phase is entered are denoted $P_{1}^{\prime}$ and $P_{2}^{\prime}$ respectively and the parameter, $g$, determines the closeness of the approximation to the step-function behaviour used previously.

Inspection of figures 20 and 21 shows how the cell phase is affected by shear-dependent proliferation. When the cell population is relatively small, disturbance to the culture medium flow is minimal and the shear remains within the proliferative region: $P_{1}^{\prime} \leqslant$ $\left|\partial p_{w} / \partial x\right| \leqslant P_{2}^{\prime}$. As the cell population increases, the increased construct density causes a reduction in $u_{w}$ near the upstream periphery, and an increase downstream (see figure 7(a)), causing the upstream shear to fall below the $P_{1}^{\prime}$ threshold and resulting in decreased proliferation there (figure 21(a)). A further increase in the cell population causes the flow disturbance to increase (see figure 7(b)) resulting in flow reversals at a number of points within the domain. This causes the shear to increase to the $P_{2}^{\prime}$ threshold and to cross the $P_{1}^{\prime}$ threshold repeatedly (see figure 21(b)), resulting in cell phase death and reduced cell phase growth at various regions within the cell population, leading to highly heterogeneous construct composition. Inspection of figures 20(b) and 21(b) shows that the influence of fluid shear stress on cell phase growth is clearest at late times. The high level of shear near the construct centre and reduced shear near the upstream periphery causes cell phase growth to be skewed in the downstream direction.

\section{Discussion}

We have presented an analysis of a multiphase model which describes tissue growth within a perfusion bioreactor, modelled as a two-dimensional channel containing a three phase mixture. The inclusion of a third phase allowed the interactions between cells and the polymer scaffold to be considered. The "cell phase" (comprising both cells and ECM) and the culture medium were modelled as viscous fluids; the formulation was greatly simplified by considering the scaffold to be spatially-homogeneous and inert. 


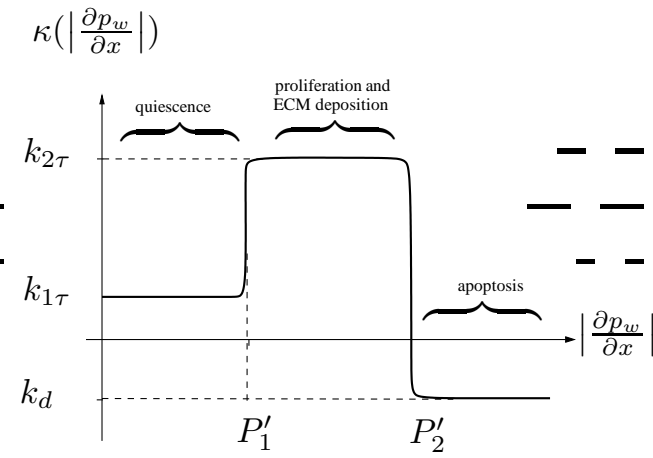

(a)

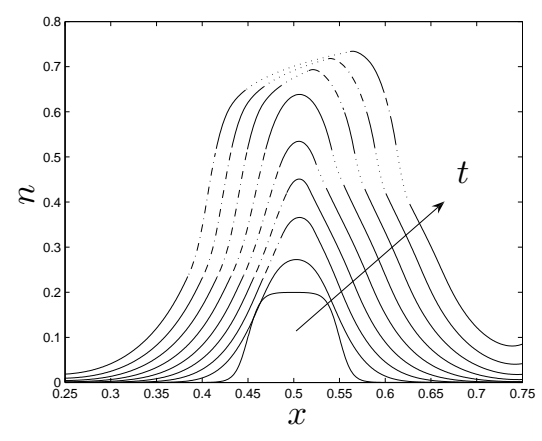

(b)

Figure 20: (a) Schematic representation of the progression of the cells from a quiescent phase to an apoptotic phase, via a proliferative phase in response to the flow-induced shear stress; (b) the evolution of the cell volume fraction, $\left|p_{w x}\right|<P_{1}^{\prime},(-.-) ; P_{1}^{\prime} \leqslant\left|p_{w x}\right| \leqslant P_{2}^{\prime}$, $(-) ;\left|p_{w x}\right|>P_{2}^{\prime},(\cdots)$, at $t=0-0.4$ (in steps of $t=0.05$ ) for growth behaviour defined by (4.3) and perfusive culture: $P_{u}=1, P_{d}=0.1, k_{m}=7.5, \bar{k}_{m}=4, k_{d}=2, D=0.01$, $\theta=0.97, P_{1}^{\prime}=0.5, P_{2}^{\prime}=1.5, g=60$.

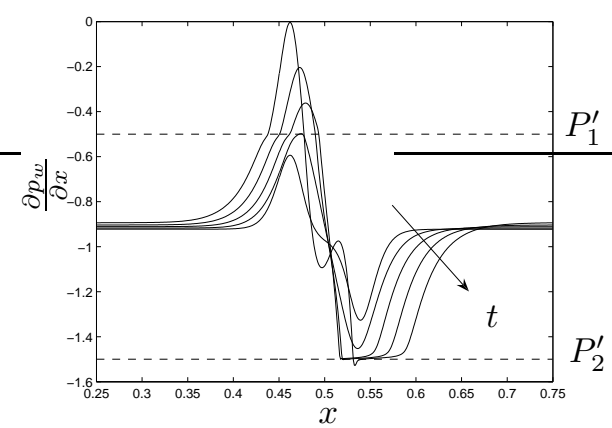

(a)

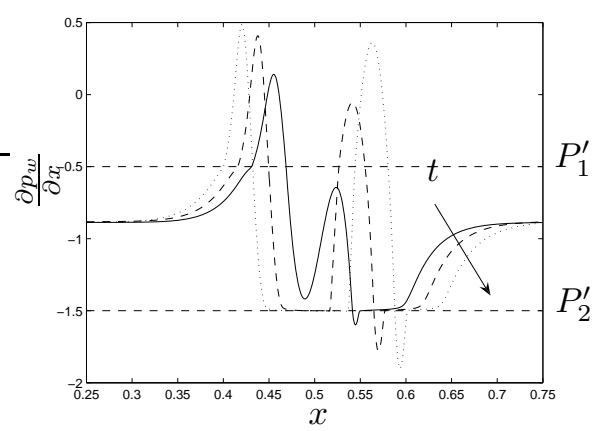

(b)

Figure 21: The evolution of the pressure gradient of the culture medium for (a) early times (small $n: t=0.02-0.22$ in steps of $t=0.05$ ), (b) later times (larger $n: t=$ $0.25,0.3 .0 .35)$, for growth behaviour defined by (4.3) and perfusive culture. Parameter values as per figure 20 .

This model represents a significant extension of the two phase model analysed by O'Dea et al. (2008) due to the retention of the individual phase variables and consideration of interphase tractions and intraphase forces, resulting in a more complex coupling 
between the dynamic culture environment and the tissue response. In contrast to O'Dea et al. (2008), our model predicted axially-symmetric growth in the absence of perfusion and showed that cell aggregation in regions of low density reduces the advection of the cell phase allowing the stringent restriction on perfusion rate implied by the large-drag analysis of O'Dea et al. (2008) to be relaxed. Furthermore, by considering cell-cell and cell-scaffold interactions, we have revealed markedly different cell behaviour depending upon the relative importance of cell aggregation and repulsion.

The numerical results and analysis presented here showed that the cell population grows, spreads and is advected downstream to a limited extent within the scaffold. Furthermore, interactions within the cell phase and between the cells and the scaffold mean that, at low cell density, the model predicts movement of cells from sparse peripheral regions to form a dense aggregate; as the density increases, repulsive interactions cause cells to be expelled from the aggregate (inspection of the model equations revealed that the cells' aggregative behaviour is dominated by cell-cell interactions). The switch between aggregative and repulsive behaviour as the cell density increases was highlighted and analysed by using a simplified form for the relevant functions: $\Sigma_{n}, \psi_{n s}$. This simple analysis showed how the diffusive behaviour of the cells is reduced or augmented depending upon the relative importance of cell aggregation and repulsion.

Analytic results, constructed in the limit of asymptotically-small cell volume fraction, take the form of a growing travelling-wave. Comparison of the predicted wave speed with that calculated from numerical simulations showed excellent quantitative agreement, and qualitative agreement was observed in the behaviour of solutions.

We further extended this model formulation to account for mechanotransduction-mediated tissue growth. This was achieved by replacing the constant growth and death rates $\left(k_{m}, k_{d}\right)$ with appropriate functional forms. To illustrate the ability of our modelling framework to accommodate mechanotransduction mechanisms relevant to a variety of tissue types, and motivated by a range of studies (see §2), we compared the response of a cell population to the local density, pressure and shear stress. Simulations were presented showing that the growth of the cell population is profoundly altered by these effects, dramatically changing the composition of the construct. These simulations clearly demonstrate the importance of considering the effect of mechanotransduction mechanisms within tissue growth models. Our model suggests that in static culture, regulation of proliferative behaviour by cell density and culture medium pressure results in indistinguishable tissue constructs; the addition of perfusion results in markedly different construct composition. In principle, on provision of appropriate experimental data, this 
conclusion provides a simple mechanism for the identification of the dominant regulatory mechanism in a given cell population. However, we note that we have not considered nutrient-limited growth which is expected to become significant in the absence of perfusion (indeed, after many days in culture, delivery of nutrients to downstream sections of the scaffold may be problematic even under perfusion, especially in scaffolds of relevant clinical thickness) and may affect the robustness of our predictions. A similar formulation in which nutrient-limited growth is considered is presented by Lemon \& King (2007); other studies which account for this include, for example, Lewis et al. (2005) and Wilson et al. (2007).

We concede that the functional forms used to model mechanotransduction-mediated growth are highly idealised and that each stimulus was considered in isolation; physiologically, these phenomena are likely to interact in a complex way to produce the cells' overall response. However, we remark that the mathematical formulation and numerical scheme developed is highly versatile, permitting the study of more complex functional forms and an investigation of the interplay between many competing growth stimuli.

We have assumed that the degradation of the rigid scaffold is negligible on the timescale of interest, corresponding to $\theta=$ constant. This greatly simplifies the three phase model equations and, in conjunction with other simplifying limits, allowed analytic progress to be made. Re-interpreting the scaffold phase as a lumped scaffold and ECM phase and introducing an equation governing its evolution provides a simple way to analyse the interplay between scaffold degradation and nascent tissue growth; such investigations are largely numerical in nature and will be presented in a subsequent study together with an investigation into the effect of choosing more biologically-relevant initial cell distributions (such as the more uniform distributions achieved via dynamic seeding on a cortical shaker (Wood et al., 2003) or peripheral seeding) and the influence of nutrient availability on the model behaviour. We emphasise that, as in the present study, since the cell phase is modelled as a viscous fluid, the predictions made in this paper will be directly relevant only to those tissue constructs whose solid characteristics are dominated by scaffold rigidity.

Lastly, we remark that we exploited the long-wavelength limit. Consequently, at leading order, the contribution of, for instance, interphase viscous drag terms are neglected from the momentum equations (2.10) and (2.11). The drag coefficient, $k$ is expected to be large (O'Dea et al., 2008) so this effect should, perhaps, be considered (we note that this may be remedied by choosing $k=\mathcal{O}\left(1 / h^{2}\right)$, where $k$ is the coefficient of viscous drag and $h$ is the channel aspect ratio). Furthermore, the dimensions of the bioreactor system are inconsistent with this limit (see figure 1). However, the bioreactor systems employed in 
tissue engineering applications are necessarily bespoke, coming in many different shapes and sizes; this analysis is particularly appropriate for those with a small aspect ratio.

\section{Acknowledgements}

We gratefully acknowledge funding from the EPSRC in the form of a studentship (R.D.O) and an Advanced Research Fellowship (S.L.W). Collaboration with A. El-Haj (ISTM, Keele University) is also acknowledged. We are grateful to Dr. Ronald Fowler (CCLRC, Rutherford Appleton Laboratory, Didcot) for help with the numerical code.

\section{A Asymptotically-small cell density: $D \neq 0$}

We now consider the solution of (3.5) and (3.6) subject to the boundary conditions (3.10) and (3.11) without additional simplification. Noting that the constant, $\gamma$ is given by equations (3.7) and (3.12), we make the following transformation:

$$
n_{1}(x, t)=e^{\left(k_{m}-k_{d}\right) t} \phi(\xi, \tau), \quad \xi=x-\gamma t, \quad \tau=D t,
$$

and we may then express equation (3.5) and its attendant boundary conditions as follows:

$$
\frac{\partial \phi}{\partial \tau}=\frac{\partial^{2} \phi}{\partial \xi^{2}}, \xi \in \Omega, \quad \frac{\partial \phi}{\partial \xi}=\widetilde{A} \phi, \text { on } \partial \Omega,
$$

where $\widetilde{A}=-A_{0} /\left[12 \mu_{n} \theta D\right]$ and the region of interest, $\xi \in[L, R]$, is denoted $\Omega$ with moving boundary $\partial \Omega ; L(\tau)=a-\gamma \tau / D$ and $R(\tau)=b-\gamma \tau / D$.

A solution to (A.2) may be constructed by considering the free-space Green's function of the simpler problem:

$$
\frac{\partial \phi}{\partial \tau}=\frac{\partial^{2} \phi}{\partial \xi^{2}}, \quad \phi( \pm \infty, \tau), \phi_{\xi}( \pm \infty, \tau) \rightarrow 0,
$$

which is found to be:

$$
G_{F}(\xi, \tau ; \eta, \mathrm{T})=\frac{H(\mathrm{~T}-\tau)}{\sqrt{4 \pi(\mathrm{T}-\tau)}} \exp \left[-\frac{(\xi-\eta)^{2}}{4(\mathrm{~T}-\tau)}\right],
$$

where $\eta$ is an arbitrary point in the domain and $\mathrm{T}>\tau$ (see, for example, Zauderer (1989)). The Green's function for the problem (A.2) can then be expressed in the form

$$
G(\xi, \tau ; \eta, \mathrm{T})=G_{F}(\xi, \tau ; \eta, \mathrm{T})+G_{B}(\xi, \tau ; \eta, \mathrm{T})
$$


where $G_{F}$ is the free-space Green's function defined above and $G_{B}$ is specified via the method of images to satisfy the boundary conditions. To satisfy the Robin boundary condition at $\xi=L(\tau)$, we consider a source point at $\xi=L(\tau)+\eta$ and introduce an image at $\Gamma=L(\tau)-\eta$ and a line of image sources extending from our image point, $\xi=\Gamma$, to $\xi=-\infty$, weighted by a density function, $\rho_{-}$, to be determined (Zauderer, 1989):

$$
G_{B}=G_{F}(\xi, \tau ; \Gamma, \mathrm{T})+\int_{-\infty}^{\Gamma} \rho_{-}(s) G_{F}(\xi, \tau ; s, \mathrm{~T}) \mathrm{d} s .
$$

In order that the condition at $\xi=R(\tau)$ is satisfied, we must add images with respect to $\xi=R(\tau)$ of the source and images points at $\xi=L(\tau) \pm \eta$ and a second line of image sources extending from the point $\xi=2 R(\tau)-L(\tau)-\eta$ (denoted $\zeta$ ) to $\xi=\infty$, weighted by a density function, $\rho_{+}(s)$, to be determined. Each of these images must, in turn, have images with respect to $L(\tau), R(\tau)$ and we are led to consider an infinite sequence of image source points and image source lines. The weighting functions for each image are determined from the boundary condition (A.2b).

The Green's function, $G$, may then be expressed:

$$
\begin{aligned}
G(\xi, \tau ; \eta, \mathrm{T})= & \frac{H(\mathrm{~T}-\tau)}{\sqrt{4 \pi(\mathrm{T}-\tau)}}\left\{\sum_{n=0}^{\infty} \exp \left[-\frac{(\xi \pm \eta-2 n R+(2 n-1) L)^{2}}{4(\mathrm{~T}-\tau)}\right]\right. \\
& -2 \widetilde{A} \int_{-\infty}^{\Gamma_{n}} \exp \left[\widetilde{A}\left(s-\Gamma_{n}\right)\right] \exp \left[-\frac{(\xi-s)^{2}}{4(\mathrm{~T}-\tau)}\right] \mathrm{d} s \\
& +\sum_{n=1}^{\infty} \exp \left[-\frac{(\xi \pm \eta+2 n R-(2 n+1) L)^{2}}{4(\mathrm{~T}-\tau)}\right] \\
& \left.-2 \widetilde{A} \int_{\zeta_{n}}^{\infty} \exp \left[\widetilde{A}\left(\zeta_{n}-s\right)\right] \exp \left[-\frac{(\xi-s)^{2}}{4(\mathrm{~T}-\tau)}\right] \mathrm{d} s\right\}
\end{aligned}
$$

wherein $\Gamma_{n}$ and $\zeta_{n}$ are defined as follows:

$$
\begin{array}{cc}
\Gamma_{n}=-2 n R+(2 n+1) L-\eta, & n=0,1,2, \ldots, \\
\zeta_{n}=2 n R-(2 n-1) L-\eta, & n=1,2,3, \ldots
\end{array}
$$

Noting that the Green's function for the problem (A.2) satisfies the following equation:

$$
-\frac{\partial G}{\partial \tau}-\frac{\partial^{2} G}{\partial \xi^{2}}=\delta(\xi-\eta) \delta(\tau-\mathrm{T}), \quad \xi, \eta \in \Omega ; \tau, \mathrm{T}<\widetilde{T}, \mathrm{~T}>0
$$

(where $\widetilde{T}$ is the temporal end point) with end and boundary conditions:

$$
G(\xi, \widetilde{T} ; \eta, \mathrm{T})=0, \quad G-\left.\widetilde{A} \frac{\partial G}{\partial \xi}\right|_{\partial \Omega}=0
$$


it can be shown, (e.g. Zauderer (1989)) that the solution, $\phi$, at an arbitrary point $(\eta, \mathrm{T})$ is given by

$$
\phi(\eta, \mathrm{T})=\int_{\partial \Omega_{0}} \phi(\xi, 0) G(\xi, 0 ; \eta, \mathrm{T}) \mathrm{d} \xi .
$$

Using initial conditions given by (3.2), equation (A.12) may be evaluated numerically and the corresponding correction to the pressure may be calculated by solving equation (3.6) subject to the boundary conditions (3.10) and (3.11). We obtain a diffusing, growing, travelling-wave solution for $n_{1}$. Numerical results omitted for concision.

\section{List of Figures}

1 The bioreactor system of El-Haj et al. (1990). . . . . . . . . . . . . . . . 6

2 Definition sketch: a two-dimensional channel of length $L^{*}$ and width $h^{*}$ containing a construct of length $b^{*}-a^{*} \ldots \ldots \ldots$. . . . . . . . 7

3 Evolution of the cell volume fraction $n$ for (a) static culture: $P_{u}=P_{d}=0$, (b) perfusion: $P_{u}=1, P_{d}=0.1$, at $t=0-0.297$ (in steps of $t=0.033$ ). Parameter values: $k_{m}=7.5, k_{d}=0.1, D=0.01, \theta=0.97, \nu=\chi=$ $0.3, \delta_{a}=\delta_{b}=0.1, \mu_{n}=1.3, a=0.25, b=0.75 \ldots \ldots \ldots$

4 Evolution of the culture medium pressure for (a) early times (small $n$ ): $t=0-0.231$ (in steps of $t=0.033$ ); and (b) later times (larger $n$ ): $t=0.25,0.27,0.29$, under perfusion. Parameter values as in figure $3(\mathrm{~b})$. .

5 Evolution of the cell pressure for (a) early times (small $n$ ): $t=0-0.13$ (in steps of $t=0.033$ ); and (b) later times (larger $n$ ): $t=0.21-0.29$ (in steps of $t=0.02$ ), under perfusion. Parameter values as in figure 3(b). . .

6 Evolution of the cell velocity at the channel centreline for (a) early times (small $n): t=0.033,0.066,0.1$; and (b) later times (larger $n$ ): $t=$ $0.2,0.23,0.25,0.27$ under perfusion. Parameter values as in figure $3(\mathrm{~b})$. .

7 Evolution of the culture medium velocity at the channel centreline for (a) early times ( small $n$ ): $t=0.033-0.165$ (in steps of $t=0.033$ ); and (b) later times (larger $n$ ): $t=0.25,0.27,0.29$, under perfusion. Parameter values as in figure $3(\mathrm{~b})$. . . . . . . . . . . . . . . . . 19

8 Evolution of the function $A(t)$. Parameter values as in figure $3(\mathrm{~b})$. . . . . 20

9 Evolution of the cell volume fraction, $n_{1}$, under perfusion at $t=0-0.2$ (in steps of $t=0.04$ ). $D=0, \delta=1$, other parameter values as in $\S 3.1$. $\quad 23$ 
10 Evolution of (a) the pressure correction, $p_{1}$ and, (b) the culture medium pressure, $p_{w}=p_{0}+\delta p_{1}$ in a magnified region within $a \leqslant x \leqslant b$, under perfusion at $t=0-0.2$ (in steps of $t=0.04$ ). Parameters as in figure 9 . .

11 (a) Comparison of the numerically-computed position of the maximum value of $n(-)$ compared to the predicted position of the travelling wave (- ) and, (b) the \% relative error between the calculated and predicted position for $\delta=1 / 25,1 / 5,1$. The arrows indicate the direction of increasing $\delta$. .

12 Schematic representation of the progression of the cells from a proliferative phase to an apoptotic phase, via an ECM-producing phase in response to the local cell density. . . . . . . . . . . . . . . . .

13 The evolution of (a) the cell volume fraction, $n<n_{1}^{\prime}$ and $n>n_{2}^{\prime}$, (); $n_{1}^{\prime} \leqslant n \leqslant n_{2}^{\prime},(\cdots)$ and, (b) the pressure of the culture medium, at $t=0-0.35$ (in steps of $t \approx 0.038$ ) for growth behaviour defined by (4.1) and perfusive culture: $P_{u}=1, P_{d}=0.1, k_{1 n}=6.5, k_{2 n}=7.5, k_{d}=1$, $D=0.01, \theta=0.97, n_{1}^{\prime}=0.4, n_{2}^{\prime}=0.6 \ldots \ldots \ldots$

14 The evolution of the pressure of the cell phase for (a) early times (small $n: t=0-0.15$, in steps of $t=0.0375$ ) and, (b) later times (larger $n$ : $t=0.2-0.35$ in steps of $t=0.05$ ), for growth behaviour defined by (4.1) and perfusive culture. Parameter values as per figure 13. . . . . . .

15 The evolution of (a) the velocity profile of the culture medium, (b) the velocity profile of the cell phase (at the channel centreline), at $t=0.2-$ 0.35 (in steps of $t=0.05$ ) for growth behaviour defined by (4.1) and perfusive culture. Parameter values as per figure 13. . . . . . . . . .

16 (a) Schematic representation of the progression of the cells from a quiescent phase to an apoptotic phase, via a proliferative phase in response to the pressure of the cell phase, $p_{n}$; (b) the evolution of the cell volume fraction at $t=0-0.28$ (in steps of $t=0.02$ ), $p_{n}>p_{n 2}^{\prime},(-.-)$; $p_{n 1}^{\prime} \leqslant p_{n} \leqslant p_{n 2}^{\prime},(-) ; p_{n}<p_{n 1}^{\prime},(\cdots)$, for growth behaviour defined by (4.2) and perfusive culture: $P_{u}=1, P_{d}=0.1, k_{1 p}=4, k_{2 p}=7.5$, $k_{d}=2, D=0.01, \theta=0.97, p_{n 1}^{\prime}=0.35, p_{n 2}^{\prime}=0.6 . \ldots . . .$.

17 The evolution of the pressure of the culture medium for (a) early times (small $n$ ): $t=0-0.2$ (in steps of $t=0.04$ ), (b) later times (larger $n$ ): $t=0.22-0.28$ (in steps of $t=0.02$ ), for growth behaviour defined by (4.2) and perfusive culture. Parameter values as per figure 16. . . . . . . 
18 The evolution of the pressure of the cell phase for (a) early times ( $\operatorname{small} n$ ): $t=0-0.12$ (in steps of $t=0.4$ ), (b) later times (larger $n$ ): $t=0.16-0.28$ (in steps of $t=0.03$ ), for growth behaviour defined by (4.2) and perfusive culture. Parameter values as per figure 16. . . . . . . . . . .

19 The evolution of the cell volume fraction at $t=0-0.3$ (in steps of $t=$ 0.033), $p_{n}<p_{n 1}^{\prime}, p_{n}>p_{n 2}^{\prime},(-) ; p_{n 1}^{\prime} \leqslant p_{n} \leqslant p_{n 2}^{\prime},(\cdots)$, for growth behaviour defined by (4.2) and static culture: $P_{u}=0=P_{d}, k_{1 p}=7.5$, $k_{2 p}=9, p_{n 1}=0, p_{n 2}^{\prime}=0.01$, other parameters as in figure 16. . . . . 32

20 (a) Schematic representation of the progression of the cells from a quiescent phase to an apoptotic phase, via a proliferative phase in response to the flow-induced shear stress; (b) the evolution of the cell volume fraction, $\left|p_{w x}\right|<P_{1}^{\prime},(-.-) ; P_{1}^{\prime} \leqslant\left|p_{w x}\right| \leqslant P_{2}^{\prime},(-) ;\left|p_{w x}\right|>P_{2}^{\prime},(\cdots)$, at $t=0-0.4$ (in steps of $t=0.05$ ) for growth behaviour defined by (4.3) and perfusive culture: $P_{u}=1, P_{d}=0.1, k_{m}=7.5, \bar{k}_{m}=4, k_{d}=2, D=0.01$, $\theta=0.97, P_{1}^{\prime}=0.5, P_{2}^{\prime}=1.5, g=60 \ldots \ldots \ldots$

21 The evolution of the pressure gradient of the culture medium for (a) early times (small $n: t=0.02-0.22$ in steps of $t=0.05$ ), (b) later times (larger $n: t=0.25,0.3 .0 .35)$, for growth behaviour defined by (4.3) and perfusive culture. Parameter values as per figure $20 \ldots$. . . . . . .

\section{References}

Araujo, R.P. and McElwain, D.L.S., 2004. A history of the study of solid tumour growth: The contribution of mathematical modelling. Bull. Math. Biol., 66(5):1039-1091.

Araujo, R.P. and McElwain, D.L.S , 2005. A mixture theory for the genesis of residual stresses in growing tissues I: A general formulation. SIAM J. of App. Math., 65(4): $1261-1284$.

Bakker, A. , Klein-Nulend, J. and Burger, E. , 2004. Shear stress inhibits while disuse promotes osteocyte apoptosis. Biochem. Biophys. Res. Comm., 320:1163-1168.

Bittig, T, Wartlick, O, Kicheva, A., González-Gaitán, M and Jülicher, F. , 2008. Dynamics of anisotropic tissue growth. New J. Phys., 10(6).

Breward, C.J.W. , Byrne, H.M. and Lewis, C.E. , 2002. The role of cell-cell interactions in a two-phase model for avascular tumour growth. J. Math. Biol., 45:125-152. 
Byrne, H.M. , King, J.R. , McElwain, D.L.S. and Preziosi, L. , 2003. A two-phase model of solid tumour growth. Appl. Math. Letters, 16:567-573.

Byrne, H.M and Preziosi, L. , 2003a. Modelling solid tumour growth using the theory of mixtures. Math. Med. Biol., 20(4):341-366.

Byrne, H.M. and Preziosi, L. , 2003b. Modelling solid tumour growth using the theory of mixtures. Math. Med. Biol., 20(4):341.

Cartmell, S.H. and El Haj, A.J., 2005. Mechanical bioreactors for tissue engineering. In Chaudhuri, J. and Al-Rubeai, M. , editors, Bioreactors for tissue engineering: Principles, Design and Operation, chapter 8, pages 193-209. Springer, Dordrecht, The Netherlands.

Chaplain, M.A.J. , Graziano, L. and Preziosi, L. , 2006. Mathematical modelling of the loss of tissue compression responsiveness and its role in solid tumour development. Math. Med. Biol., 23(3):197.

Coletti, F. , Macchietto, S. and Elvassore, N. , 2006. Mathematical modeling of threedimensional cell cultures in perfusion bioreactors. Ind. \& Eng. Chem. Res., 45(24): 8158-8169.

Cowin, S.C. , 2000. How is a tissue built? J. Biomech. Eng., 122:553.

Cowin, S.C. , 2004. Tissue growth and remodeling. Ann. Rev. of Biomed. Eng., 6(1): 77-107.

Curtis, A. and Riehle, M. , 2001. Tissue engineering: the biophysical background. Phys. in MEd. and Biol, 46:47-65.

Drew, D.A. , 1983. Mathematical modelling of two-phase flow. Ann. Rev. Fluid Mech., 15:261-291.

Drew, D.A. and Segel, L.A., 1971. Averaged equations for two-phase flows. Studies in Appl. Math., 50:205-231.

El-Haj, A.J. , Minter, S.L. , Rawlinson, S.C. , Suswillo, R. and Lanyon, L.E. , 1990. Cellular responses to mechanical loading in vitro. J. Bone and Min. Res., 5(9):923-32.

Franks, S.J. . 2002. Mathematical modelling of tumour growth and stability. PhD thesis, University of Nottingham, UK. 
Franks, S.J. , Byrne, H.M. , King, J.R. , Underwood, J.C.E. and Lewis, C.E. , 2003. Modelling the early growth of ductal carcinoma in situ of the breast. J. Math. Biol., 47: 424-452.

Franks, S.J. and King, J.R. , 2003. Interactions between a uniformly proliferating tumour and its surroundings: uniform material properties. Math. Med. Biol., 20:47-89.

Freyria, A.M. , Yang, Y. , Chajra, H. , Rousseau, C.F. , Ronziere, M.C. , Herbage, D. and El Haj, A.J. , 2005. Optimization of dynamic culture conditions: Effects on biosynthetic activities of chondrocytes grown in collagen sponges. Tissue Eng., 11(5-6):674-684.

Fung, Y.C., 1991. What are residual stresses doing in our blood vesels? Ann. Biomed. Eng., 19:237-249.

Han, Y. , Cowin, S.C. , Schaffler, M.B. and Weinbaaum, S. , 2004. Mechanotransduction and strain amplification in osteocyte cell processes. Proc. Nat. Acad. of Sci., 101(47): 16689-16694.

Haskin, C. , Cameron, I. and Athanasiou, K. , 1993. Physiological levels of hydrostatic pressure alter morphology and organization of cytoskeletal and adhesion proteins in MG-63 osteosarcoma cells. Biochem. Cell Biol., 71(1-2):27-35.

Jaecques, S.V.N., Van-Oosterwyck, H. , Muraru, L. , Van-Cleynbreugel, T. , De-Smet, E. , Wevers, M. , Naert, I. and Vander-Sloten, J. , 2004. Individualised, microCT-based finite element modelling as a tool for biomechanical analysis relating to tissue engineering of bone. Biomat., 25:1683-1696.

King, J.R. and Franks, S.J. , 2004. Mathematical analysis of some multi-dimensional tissue growth models. Eur. J. Appl. Math., 15(3):273-295.

Klein-Nulend, J. , Helfrich, M.H. , Sterck, J.G.H. , Macpherson, H. , Joldersma, M. , Ralston, S.H. , Semeins, C.M. and Burger, E.H. , 1998. Nitric oxide response to shear stress by human bone cell cultures is endothelial nitric oxide synthase dependent. Biochem. and Biophys. Res. Comm., 250:108-114.

Klein-Nulend, J. , Roelofsen, J., Sterck, J.G. , Semeins, C.M. and Burger, E.H. , 1995a. Mechanical loading stimulates the release of transforming growth factor-beta activity by cultured mouse calvariae and periosteal cells. J. Cell Phys., 163(1):115-119. 
Klein-Nulend, J. , Van Der Plas, A. , Semeins, C.M. , Ajubi, N.E. , Frangos, J.A. , Nijweide, P.J. and Burger, E.H. , 1995b. Sensitivity of osteocytes to biomechanical stress in vitro. FASEB, 9:441-445.

Kolev, N.I. , 2002. Multiphase Flow Dynamics, volume 1 - Fundamentals. SpringerVerlag, Berlin, Heidelberg, New York.

Landman, K.A. and Please, C.P. , 2001. Tumour dynamics and necrosis: Surface tension and stability. IMA J. of Math. Appl. in Med. and Biol., 18(2):131-158.

Lemon, G. and King, J.R. , 2007. Multiphase modelling of cell behaviour on artificial scaffolds: effects of nutrient depletion and spatially nonuniform porosity. Math. Med. Biol., 24(1):57.

Lemon, G. , King, J.R , Byrne, H.M. , Jensen, O.E. and Shakesheff, K. , 2006. Multiphase modelling of tissue growth using the theory of mixtures. J. Math. Biol., 52(2):571-594.

Lewis, M.C. , Macarthur, B.D. , Malda, J. , Pettet, G. and Please, C.P. , 2005. Heterogeneous proliferation within engineered cartilaginous tissue: the role of oxygen tension. Biotech. and Bioeng., 91(5):607-15.

Malda, J. , Rouwkema, J. , Martens, DE , le Comte, EP , Kooy, FK , Tramper, J. , van Blitterswijk, CA and Riesle, J., 2004. Oxygen gradients in tissue-engineered Pegt/Pbt cartilaginous constructs: Measurement and modelling. Biotech. and Bioeng., 86(1): 9-18.

Martin, I. , Wendt, D. and Heberer, M. , 2004. The role of bioreactors in tissue engineering. Trends in Biotechn., 22(2):80-86.

McGarry, J.G. , Klein-Nulend, J. , Mullender, M.G. and Prendergast, P.J. , 2004. A comparison of strain and fluid shear stress in simulating bone cell responses - a computation and experimental study. FASEB, 18(15).

O'Dea, R.D. . 2008. Multiphase modelling of tissue growth in dynamic culture conditions. $\mathrm{PhD}$ thesis, University of Nottingham, UK.

O’Dea, R.D. , Waters, S.L. and Byrne, H.M. , 2008. A two-fluid model for tissue growth within a dynamic flow environment. Accepted for publication in the Eur. J. Appl. Math. 
Owan, I. , Burr, D.B. , Turner, C.H. , Qiu, J. , Tu, Y. , Onyia, J.E. and Duncan, R.L. , 1997. Mechanotransduction in bone: osteoblasts are more responsive to fluid forces than mechanical strain. Am. J. Phys.: Cell Phys., 273(3):810-815.

Peirce, SM , Skalak, TC and Papin, JA , 2006. Multiscale biosystems integration: Coupling intracellular network analysis with tissue-patterning simulations. Systems Biol., 50(6).

Peregrine, D.H. , 1967. Long waves on a beach. J. Fluid Mech., 27:815-827.

Porter, B. , Zauel, R. , Stockman, H. , Guldberg, R. and Fyhrie, D. , 2005. 3-D computational modeling of media flow through scaffolds in a perfusion bioreactor. J. Biomech., 38(3):543-549.

Raimondi, M.T. , 2004. The effect of media perfusion on three-dimensional cultures of human chondrocytes: Integration of experimental and computational approaches. Biorheology, 41(3):401-410.

Roelofsen, J. , Klein-Nulend, J. and Burger, E.H. , 1995. Mechanical stimulation by intermittent hydrostatic compression promotes bone-specific gene expression in vitro. J. Biomech., 28(12):1493-1503.

Roose, T. , Neti, P.A. , Munn, L.L., Boucher, Y. and Jain, R.K. , 2003. Solid stress generated by spheroid growth estimated using a poroelasticity model. Microvascular Res., 66:204-212.

Sipe, J.D. , 2002. Tissue Engineering and Reparative Med. Ann. of the New York acad. of Sci., 961:1-9.

Tracqui, P. and Ohayon, J. , 2004. Transmission of mechanical stresses within the cytoskeleton of adgerent cells: a theoretical analysis based on a multi-component cell model. Acta Biotheoretica, 52:323-341.

Weinbaum, S. , Cowin, S.C. and Zeng, Y. , 1994. A model for the excitation of osteosytes by mechanical loading-induced bone fluid shear stresses. J. Biomech., 27(3):339-360.

Wilson, DJ , King, JR and Byrne, HM , 2007. Modelling scaffold occupation by a growing, nutrient-rich tissue. Math. Models and Methods in Appl. Sci., 17:1721.

Wood, MA, Marshall, G. , Yang, Y. and El Haj, A. , 2003. Seeding efficiency and distribution of primary osteoblasts in 3D porous poly (L-lactide) scaffolds. Eur. Cells and Mat., 6:58. 
Wood, M.A. , Yang, Y. , Thomas, P.B.M. and Haj, A.J.E. , 2006. Using DihydropyridineRelease Strategies to Enhance Load Effects in Engineered Human Bone Constructs. Tissue Eng., 12(9):2489-2497.

You, J., Yellowley, C.E., Donahue, H.J., Zhang, Y., Chen, Q. and Jacobs, C.R., 2000. Substrate deformation levels associated with routine physical activity are less stimulatory to bone cells relative to loading-induced oscillatory fluid flow. J. Biomech. Eng., 122:377-393.

You, L., Cowin, S.C., Schaffler, M.B. and Weinbaum, S. , 2001. A model for strain amplification in the actin cytoskeleton of osteocytes due to fluid drag on pericellular matrix. J. Biomech., 34(11):1375-86.

Zauderer, E. , 1989. Partial Differential Equations of Applied Mathematics. Wiley Interscience, second edition. 